\title{
On the Rayleigh-Taylor Instability for Two Uniform Viscous Incompressible Flows
}

\author{
Fei Jiang, ${ }^{\mathrm{a}, *}$, Song Jiang ${ }^{\mathrm{a}}$, Weiwei Wang ${ }^{\mathrm{b}}$ \\ ${ }^{a}$ Institute of Applied Physics and Computational Mathematics, Beijing, 100088, China. \\ ${ }^{b}$ School of Mathematical Sciences, Xiamen University, Xiamen 361005, China
}

\begin{abstract}
We study the Rayleigh-Taylor instability for two incompressible immiscible fluids with or without surface tension, evolving with a free interface in the presence of a uniform gravitational field in Eulerian coordinates. To deal with the free surface, instead of using the transform of Lagrangian coordinates, we transform the perturbed equations in Eulerian coordinates to an integral form and formulate the two-fluids flow in a single-fluid flow in a fixed domain, thus offering an alternative approach to deal with the jump conditions at the free interface. First, we analyze the linearized problem around the steady state which describes a denser immiscible fluid lying above a light one with an free interface separating the two fluids, and both fluids being in (unstable) equilibrium. By a general method of studying a family of modes, we construct smooth (when restricted to each fluid domain) solutions to the linearized problem that grow exponentially fast in time in Sobolev spaces, thus leading to an global instability result for the linearized problem. Then, using these pathological solutions, we demonstrate the global instability for the corresponding nonlinear problem in an appropriate sense.
\end{abstract}

Keywords: Rayleigh-Taylor instability, viscous incompressible flows, global instability.

\section{Introduction}

We consider the two-phase free boundary problem for the equations of two incompressible immiscible fluids within the infinite slab $\Omega=\mathbb{R}^{2} \times(-1,1) \subset \mathbb{R}^{3}$ and for time $t \geq 0$. The fluids are separated by a moving free interface $\Sigma(t)$ which is given by the unknown function $\eta: \mathbb{R}^{+} \times \mathbb{R}^{2} \rightarrow \mathbb{R}$. Hence we can definite $\Sigma(t):=\left\{x \in \mathbb{R}^{3} \mid x_{3}=\eta\left(t, x^{\prime}\right)\right\}$ for each $t \geq 0$, where $x^{\prime}=\left(x_{1}, x_{2}\right)^{T}$, and the superscript $T$ means matrix transposition.

The interface divides $\Omega$ into two time-dependent, disjoint, open subsets $\Omega_{ \pm}(t)$, so that $\Omega=$ $\Omega_{+}(t) \cup \Omega_{-}(t) \cup \Sigma(t)$ and $\Sigma(t)=\bar{\Omega}_{+}(t) \cap \bar{\Omega}_{-}(t)$. The motion of the fluids is driven by the constant gravitational field along $e_{3}$-the $x_{3}$ direction, $G=(0,0,-g)^{T}$ with $g>0$. The two fluids are described by their velocity, and pressure functions, which are given for each $t \geq 0$ by

$$
\left(u_{ \pm}, \bar{p}_{ \pm}\right)(t, \cdot): \Omega_{ \pm}(t) \rightarrow\left(\mathbb{R}^{3}, \mathbb{R}^{+}\right),
$$

respectively. We assume that at a given time $t \geq 0$, these functions have well-defined traces onto $\Sigma(t)$.

The fluids under consideration are incompressible and viscous. Hence, for $t>0$, the fluids satisfy the following motion equations:

\footnotetext{
*Corresponding author: Tel +86 15001201710 .

Email addresses: jiangfei0591@163.com (Fei Jiang), jiang@iapcm.ac.cn (Song Jiang), wei.wei.840163.com (Weiwei Wang)
} 


$$
\left\{\begin{array}{lr}
\partial_{t} \eta=u_{3}-u_{1} \partial_{1} \eta-u_{2} \partial_{2} \eta, & \text { on } \Sigma(t), \\
\partial_{t}\left(\varrho_{ \pm} u_{ \pm}\right)+\operatorname{div}\left(\varrho_{ \pm} u_{ \pm} \otimes u_{ \pm}\right)+\operatorname{div} S_{ \pm}=-g \varrho_{ \pm} e_{3}, & \text { in } \Omega_{ \pm}(t), \\
\operatorname{div} u_{ \pm}=0, & \text { in } \Omega_{ \pm}(t),
\end{array}\right.
$$

where $(1.1)_{1}$ describes the motion of the free interface (cf. [7]), $\partial_{i}:=\partial_{x_{i}}$, the positive constants $\varrho_{ \pm}$denote the densities of the respective fluids, and we define the stress tensor by

$$
S_{ \pm}=-\mu_{ \pm}\left(\nabla u_{ \pm}+\nabla u_{ \pm}^{T}\right)+\bar{p}_{ \pm} I .
$$

with $\mu_{ \pm}$and $\bar{p}_{ \pm}$being the viscosity coefficient and the pressure of the respective fluids, and $I$ the $3 \times 3$ identity matrix.

For two viscous fluids meeting at a free boundary with surface tension, from the physical point of view, the velocity is continuous across the interface and the jump in the normal stress is proportional to the mean curvature of the surface multiplied by the normal to the surface (cf. $[2,19])$. Thus, we impose the jump conditions at the free interface:

$$
\begin{aligned}
& {\left.[u]\right|_{\Sigma(t)}=0,} \\
& {\left.[S \nu]\right|_{\Sigma(t)}=\kappa H \nu,}
\end{aligned}
$$

where the interfacial jump is defined by

$$
\left.[f]\right|_{\Sigma(t)}:=\left.f_{+}\right|_{\Sigma(t)}-\left.f_{-}\right|_{\Sigma(t)},
$$

$\left.f\right|_{\Sigma(t)}$ is the trace of a quantity $f$ on $\Sigma(t)$, and

$$
\nu=\frac{\left(-\partial_{1} \eta,-\partial_{2} \eta, 1\right)^{T}}{\sqrt{1+\left(\partial_{1} \eta\right)^{2}+\left(\partial_{2} \eta\right)^{2}}}
$$

denotes the normal vector to the free surface $\Sigma(t)$. The jump condition of (1.2) implies that there is no possibility of the fluids slipping past each other along $\Sigma(t)$. Here we take $H$ to be twice the mean curvature of the surface $\Sigma(t)$ and the surface tension to be a constant $\kappa \geq 0$. Since $\Sigma(t)$ is parameterized by $\left(x^{\prime}, \eta\left(t, x^{\prime}\right)\right)$, we may employ the standard formula for the mean curvature of a parameterized surface to write

$$
H=\frac{\Delta_{x^{\prime}} \eta+\left(\partial_{1} \eta\right)^{2} \partial_{2}^{2} \eta+\left(\partial_{2} \eta\right)^{2} \partial_{1}^{2} \eta-2 \partial_{1} \eta \partial_{2} \eta \partial_{1} \partial_{2} \eta}{\left(1+\left(\partial_{1} \eta\right)^{2}+\left(\partial_{2} \eta\right)^{2}\right)^{3 / 2}}
$$

We also enforce the condition that the normal component of the fluid velocity vanishes at the fixed boundaries, that is,

$$
u_{+}\left(t, x^{\prime},-1\right)=u_{-}\left(t, x^{\prime}, 1\right)=0, \quad \text { for all } t \geq 0, x^{\prime} \in \mathbb{R}^{2} .
$$

To complete the statement of the problem, we have to specify initial conditions. We give the initial interface $\Sigma(0)=\Sigma_{0}$, which yields the open sets $\Omega_{ \pm}(0)$ on which we specify the initial data for the velocity and height of interface

$$
u_{ \pm}(0, \cdot): \Omega_{ \pm}(0) \rightarrow \mathbb{R}^{3}, \quad \eta(0, \cdot): \mathbb{R}^{2} \rightarrow(-1,1) .
$$

Thus the initial datum of the pressure can be given by $\varrho_{ \pm}, \eta(0, \cdot)$ and $u_{ \pm}(0, \cdot)$. To simply the equations, we introduce the indicator functions $\chi_{\Omega_{ \pm}}$and denote

$$
\varrho=\varrho_{+} \chi_{\Omega_{+}}+\varrho_{-} \chi_{\Omega_{-}}, u=u_{+} \chi_{\Omega_{+}}+u_{-} \chi_{\Omega_{-}}, \bar{p}=\bar{p}_{+} \chi_{\Omega_{+}}+\bar{p}_{-} \chi_{\Omega_{-}},
$$


to define the modified pressure by

$$
p=\bar{p}+g \varrho x_{3} .
$$

Thus, for each $t>0$, the equations (1.1) can be rewritten as

$$
\begin{cases}\partial_{t} \eta=u_{3}-u_{1} \partial_{1} \eta-u_{2} \partial_{2} \eta, & \text { on } \Sigma(t), \\ \varrho \partial_{t} u+\varrho(\nabla u) u+\nabla p=\mu \Delta u, & \text { in } \Omega \backslash \Sigma(t), \\ \operatorname{div} u=0, & \text { in } \Omega \backslash \Sigma(t)\end{cases}
$$

and the jump condition (1.3) becomes, setting $[\varrho]=\varrho_{+}-\varrho_{-}$,

$$
\left.\left[\left(p I-\mu\left(\nabla u+\nabla u^{T}\right)\right) \nu\right]\right|_{\Sigma(t)}=(g[\varrho] \eta+\kappa H) \nu .
$$

For convenience in the subsequent analysis, we will use the natation

$$
\llbracket f \rrbracket:=\left.f_{+}\right|_{x_{3}=0}-\left.f_{-}\right|_{x_{3}=0}
$$

for the jump of a quantity $f$ across the set $\left\{x_{3}=0\right\}$.

Now, we linearize the equations (1.4) around a steady-state solution $\eta=0, u=0$ and $p=$ constant, then the resulting linearized equations read as

$$
\begin{cases}\partial_{t} \eta=u_{3}, & \text { on } \mathbb{R}^{+} \times\left\{x_{3}=0\right\}, \\ \varrho \partial_{t} u+\nabla p=\mu \Delta u, & \text { in } \mathbb{R}^{+} \times\left(\Omega \backslash\left\{x_{3}=0\right\}\right), \\ \operatorname{div} u=0, & \text { in } \mathbb{R}^{+} \times\left(\Omega \backslash\left\{x_{3}=0\right\}\right) .\end{cases}
$$

The corresponding linearized jump conditions are

$$
\llbracket u \rrbracket=0, \llbracket p I-\mu\left(\nabla u+\nabla u^{T}\right) \rrbracket e_{3}=\left(g[\varrho] \eta+\kappa \Delta_{x^{\prime}} \eta\right) e_{3},
$$

while the boundary conditions are

$$
u\left(t, x^{\prime},-1\right)=u\left(t, x^{\prime}, 1\right)=0 .
$$

Considering two completely plane-parallel layers of immiscible fluid, the heavier on top of the light one and both subject to the earth's gravity. In this case, the equilibrium state is unstable to sustain small perturbations or disturbances, and this unstable disturbance will grow and lead to a release of potential energy, as the heavier fluid moves down under the (effective) gravitational field, and the lighter one is displaced upwards. This phenomena was first studied by Rayleigh $[14,15]$ and then Taylor [16], and therefore, is called the Rayleigh-Taylor instability. In the last decades, a lot of works related to this phenomena have been made from both physical and numerical points of view. In particular, many results concerning linearized problems have been summarized in monographs, see, for example, $[2,17]$. To our best knowledge, however, there are only few mathematical analysis results on nonlinear problems in the literature, due to the fact that in general, passage from a linearized instability to a dynamical nonlinear instability for a conservative nonlinear partial differential system is rather difficult. In 1987, Ebin [4] proved the ill-posedness of the equations of motion for a perfect fluid with free boundary. Then, he adapted the approach of [4] to obtain the ill-posedness of both Rayleigh-Taylor and Helmholtz problems for two-dimensional incompressible, immiscible, inviscid fluids without surface tension [5]. In 2003, Hwang and Guo [10] showed the nonlinear Rayleigh-Taylor instability for twodimensional, incompressible, inviscid fluids with continuous density, and their result was extended to magnetohydrodynamic (MHD) flows [9] recently. Unfortunately, the approaches in both [5] 
and [10] could not be applied to the viscous flow case, since the viscosity can bring some technical difficulties in the study of the nonlinear Rayleigh-Taylor instability.

In 2011, for two-compressible immiscible fluids evolving with a free interface (the density is discontinuous across the free interface), Y. Guo and I. Tice made use of flow maps (Lagrangian coordinates) to transfer the free boundary into a fixed boundary and established a variational framework for nonlinear instability in [6], where with the help of the method of Fourier synthesis, they constructed solutions that grow arbitrarily quickly in time in the Sobolev space, leading to the ill-posedness of the perturbed problem in Lagrangian coordinates. It should be noted that they also investigated the stabilized effect of viscosity and surface tension to the linear RayleighTaylor instability (see [8]), however, the nonlinear instability still remains open.

In this paper, we will study the nonlinear Rayleigh-Taylor instability for two unform viscous incompressible flows with surface tension and a free interface, across which the density is discontinuous. We will prove that in Eulerian coordinates, the corresponding linearized system is globally unstable in the sense of Hadamard, and moreover, the original nonlinear problem with or without surface tension is globally unstable. For this purpose, we assume that $\kappa \geq 0$ and that the upper fluid is heavier than the lower fluid, i.e.

$$
\varrho_{+}>\varrho_{-} \Leftrightarrow[\varrho]>0
$$

We mention that the analogue of the Rayleigh-Taylor instability arises when the fluids are electrically conducting and a magnetic field is present, and the growth of the instability will be influenced by the magnetic field due to the generated electromagnetic induction and the Lorentz force $[3,9,11,18]$. Some authors have extended the partial results concerning the Rayleigh-Taylor instability of superposed flows to the case of MHD flows by overcoming additional difficulties induced by presence of the magnetic field.

This paper is organized as follows. In Section 2 we state our results on the linearized system (1.5) and nonlinear system (1.4), i.e., Theorems 2.1 and 2.2. In Section 3 we construct the growing solutions to the linearized equations, while in Section 4 we analyze the linear problem and prove the uniqueness and Theorem 2.1. In Section 5, we prove the global instability of order $k$ of the nonlinear problem, i.e. Theorem 2.2.

\section{Main results}

Before stating the main results, we introduce the notation that will be used throughout the paper. For a function $f \in L^{2}(\Omega)$, we define the horizontal Fourier transform via

$$
\hat{f}\left(\xi, x_{3}\right)=\int_{\mathbb{R}^{2}} f\left(x^{\prime}, x_{3}\right) e^{-i x^{\prime} \cdot \xi} \mathrm{d} x^{\prime},
$$

where $x^{\prime}, \xi \in \mathbb{R}^{2}$, and "." denotes scalar product. By the Fubini and Parseval theorems, we have that

$$
\int_{\Omega}|f(x)|^{2} \mathrm{~d} x=\frac{1}{4 \pi^{2}} \int_{\Omega}\left|\hat{f}\left(\xi, x_{3}\right)\right|^{2} \mathrm{~d} \xi \mathrm{d} x_{3} .
$$

We now define a function space suitable for our analysis of two disjoint fluids. For a function $f$ defined on $\Omega$ we write $f_{+}$for the restriction to $\Omega_{+}=\mathbb{R}^{2} \times(0,1)$ and $f_{-}$for the restriction to $\Omega_{-}=\mathbb{R}^{2} \times(-1,0)$. For $s \in \mathbb{R}$, we define the piecewise Sobolev space of order $s$ by

$$
H^{s}\left(\Omega_{ \pm}\right)=\left\{f \mid f_{+} \in H^{s}\left(\Omega_{+}\right), f_{-} \in H^{s}\left(\Omega_{-}\right)\right\}
$$

endowed with the norm

$$
\|f\|_{H^{s}\left(\Omega_{ \pm}\right)}^{2}=\|f\|_{H^{s}\left(\Omega_{+}\right)}^{2}+\|f\|_{H^{s}\left(\Omega_{-}\right)}^{2} .
$$


Similarly to $(2.1)$, for a function $f$ defined on $(0, \infty) \times \Omega$, for which an interface divides $\Omega$ into two time-dependent, disjoint, open subsets $\Omega_{ \pm}(t)$, so that $\Omega=\Omega_{+}(t) \cup \Omega_{-}(t) \cup \Sigma(t)$ and $\Sigma(t)=\bar{\Omega}_{+}(t) \cap \bar{\Omega}_{-}(t)$, we denote

$$
H^{s}\left(\Omega_{ \pm}(t)\right)=\left\{f(t) \mid f_{+}(t) \in H^{s}\left(\Omega_{+}(t)\right), f_{-}(t) \in H^{s}\left(\Omega_{-}(t)\right)\right\}
$$

for each $t \in[0, \infty)$.

In addition, for $k \in \mathbb{N}$ we can take the norms to be given by

$$
\begin{aligned}
\|f\|_{H^{k}\left(\Omega_{ \pm}\right)}^{2} & :=\sum_{j=0}^{k} \int_{\mathbb{R}^{2} \times I_{ \pm}}\left(1+|\xi|^{2}\right)^{k-j}\left|\partial_{x_{3}}^{j} \hat{f}_{ \pm}\left(\xi, x_{3}\right)\right|^{2} \mathrm{~d} \xi \mathrm{d} x_{3} \\
& =\sum_{j=0}^{k} \int_{\mathbb{R}^{2}}\left(1+|\xi|^{2}\right)^{k-j}\left\|\partial_{x_{3}}^{j} \hat{f}_{ \pm}(\xi, \cdot)\right\|_{L^{2}\left(I_{ \pm}\right)}^{2} \mathrm{~d} \xi
\end{aligned}
$$

where $I_{-}=(-1,0)$ and $I_{+}=(0,1)$. The main difference between the piecewise Sobolev space $H^{s}(\Omega)$ and the usual Sobolev space lies in that we do not require functions in the piecewise Sobolev space to have weak derivatives across the set $\left\{x_{3}=0\right\}$. If $f:=\left(f_{1}, \cdots, f_{n}\right)^{T} \in$ $\left(H^{s}\left(\Omega_{ \pm}\right)\right)^{n}$, to shorten notation, we define

$$
\|f\|_{H^{s}\left(\Omega_{ \pm}\right)}^{2}=\sum_{i=1}^{n}\left\|f_{i}\right\|_{H^{s}\left(\Omega_{ \pm}\right)}^{2} .
$$

Now, we are in a position to state our first result, i.e. the result of global instability for the linearized problem (1.5).

Theorem 2.1. The linearized problem (1.5) with the corresponding jump and boundary conditions is globally unstable in the sense of Hadamard in $H^{k}(\Omega)$ for every $k$. More precisely, there exists a constant $C_{1}>0$, and for any $k, j \in \mathbb{N}$ with $j \geq k$ and for any $\alpha>0$, there exist a constant $C_{j, k}$ depending on $j$ and $k$, and a sequence of solutions $\left\{\left(\eta_{n}, u_{n}, p_{n}\right)\right\}_{n=1}^{\infty}$ to (1.5) satisfying the corresponding jump and boundary conditions (1.6), (1.7), so that

$$
\left\|\eta_{n}(0)\right\|_{H^{j}\left(\mathbb{R}^{2}\right)}+\left\|u_{n}(0)\right\|_{H^{j}\left(\Omega_{ \pm}\right)}+\left\|p_{n}(0)\right\|_{H^{j}\left(\Omega_{ \pm}\right)} \leq \frac{1}{n}
$$

but

$$
\left\|u_{n}(t)\right\|_{H^{k}\left(\Omega_{ \pm}\right)} \geq \alpha \text { for all } t \geq t_{n}:=C_{j, k}+C_{1} \ln (\alpha n) .
$$

Moreover,

$$
\left\|u_{n}(t)\right\|_{H^{k}\left(\Omega_{ \pm}\right)} \rightarrow \infty \text { as } t \rightarrow \infty .
$$

Theorem 2.1 shows globally discontinuous dependence of solutions upon initial data. The proof of Theorem 2.1 is inspired by [8] under necessary modifications and its basic idea is the following. First, we notice that the linearized equations have coefficients that depend only on the vertical variable $x_{3} \in(-1,1)$. This allows us to seek "normal mode" solutions by taking the horizontal Fourier transform of the equations and assuming that the solutions grow exponentially in time by the factor $e^{\lambda(|\xi|) t}$, where $\xi \in \mathbb{R}^{2}$ is the horizontal spatial frequency and $\lambda(|\xi|)>0$. This reduces the equations to a system of ordinary differential equations with $\lambda(|\xi|)>0$ for each $\xi$. Then, solving the ODE system by the modified variational method, we show that $\lambda(|\xi|)>0$ is a continuous function on $\left(0,|\xi|_{c}\right)$, the normal modes with spatial frequency grow in time, providing a mechanism for the Rayleigh-Taylor global instability, where $|\xi|_{c}=\sqrt{g[\varrho] / \kappa}$ if $\kappa>0$, otherwise $|\xi|_{c}=\infty$. Indeed, we can restrict $\xi$ in some annulus domain such that $\lambda(|\xi|)$ has a uniformly 
lower bound, then we form a Fourier synthesis of the normal mode solutions constructed for each spatial frequency $\xi$ to give solutions of the linearized incompressible equations that grow in time, when measured in $H^{k}(\Omega)$ for any $k \geq 0$. Finally, we exploit the property of boundary trace theorem to show a uniqueness result of the linearized problem (i.e. Theorem 4.1), with the help of which we obtain the global instability of the corresponding nonlinear problem (i.e. Theorem 2.2 ). In spite of the uniqueness, the linearized problem is globally unstable in $H^{k}(\Omega)$ for any $k$ in the sense of Hadamard.

With the linear global instability established, we can show the global instability of the corresponding nonlinear problem in some sense. Recalling that the steady state solution to (1.4) is given by $\eta=0, u=0, p=$ constant, we now rewrite the nonlinear equations (1.4) in the form of perturbation around the steady state. Let

$$
\eta=0+\eta, u=0+u, p=\text { constant }+\sigma .
$$

Then, the system (1.4) can be rewritten for $(\eta, u, \sigma)$ as

$$
\left\{\begin{array}{l}
\partial_{t} \eta=u_{3}-u_{1} \partial_{1} \eta-u_{2} \partial_{2} \eta \\
\varrho \partial_{t} u+\varrho(\nabla u) u+\nabla \sigma=\mu \Delta u \\
\operatorname{div} u=0
\end{array}\right.
$$

The jump conditions across the interface are

$$
\begin{aligned}
& {\left.[u]\right|_{\sum(t)}=0,} \\
& {\left.\left[\left(\sigma I-\mu\left(\nabla u+\nabla u^{T}\right)\right) \nu\right]\right|_{\sum(t)}=(g[\varrho] \eta+\kappa H) \nu,}
\end{aligned}
$$

where

$$
\Sigma(t):=\left\{x \in \mathbb{R}^{3} \mid x_{3}=\eta\left(t, x^{\prime}\right)\right\} \subset \Omega \text { for each } t \geq 0 .
$$

Finally, we require the boundary condition

$$
u_{-}\left(t, x^{\prime},-1\right)=u_{+}\left(t, x^{\prime}, 1\right)=0 .
$$

We collectively refer to the evolution, jump, and boundary equations (2.6)-(2.9) as "the perturbed problem".

Definition 2.1. We say that the perturbed problem has global stability of order $k$ for some $k \geq 3$ if there exist $\delta, C_{2}>0$ and a function $F:[0, \delta) \rightarrow \mathbb{R}^{+}$satisfying $F(z) \leq C_{2} z$ for $z \in[0, \delta)$, so that the following holds. For any $\eta_{0}, u_{0}$ satisfying

$$
\left\|\eta_{0}\right\|_{H^{k}\left(\mathbb{R}^{2}\right)}+\left\|u_{0}\right\|_{H^{k}\left(\Omega_{ \pm}(0)\right)}<\delta
$$

there exist $\eta(t) \in H^{2}\left(\mathbb{R}^{2}\right) \cap C_{\text {loc }}^{0,1}\left(\mathbb{R}^{2}\right), u(t) \in\left(H^{3}\left(\Omega_{ \pm}(t)\right) \cap C^{0}(\bar{\Omega}) \cap H_{0}^{1}(\Omega)\right)^{3}$ and $\sigma \in H^{1}\left(\Omega_{ \pm}(t)\right)$ for any $t \geq 0$, so that

(1) $(\eta, u)(0)=\left(\eta_{0}, u_{0}\right)$

(2) $\eta, u, \sigma$ solve the perturbed problem (2.6)-(2.9),

(3) $\eta \in C^{0}\left([0,+\infty), L_{\mathrm{loc}}^{2}\left(\mathbb{R}^{2}\right)\right)$, and $u \in C^{0}\left([0,+\infty),\left(L^{2}(\Omega)\right)^{3}\right)$,

(4) it holds that

$$
\sup _{0 \leq t<+\infty}\left(\|u\|_{H^{3}\left(\Omega_{ \pm}(t)\right)}+\|\eta\|_{H^{2}\left(\mathbb{R}^{2}\right)}+\|\sigma\|_{H^{1}\left(\Omega_{ \pm}(t)\right)}\right) \leq F\left(\left\|\eta_{0}\right\|_{H^{k}\left(\mathbb{R}^{2}\right)}+\left\|u_{0}\right\|_{H^{k}\left(\Omega_{ \pm}(0)\right)}\right) .
$$


We can show that the property of global stability of order $k$ cannot hold for any $k \geq 3$, i.e. the following Theorem 2.1, which will be proved in Section 5 .

Theorem 2.2. The perturbed problem does not have property of global stability of order $k$ for any $k \geq 3$.

The basic idea in the proof of Theorem 2.2 is to show, by utilizing the Lipschitz structure of $F$, that the global stability of order $k$ would give rise to certain estimates of solutions to the linearized equations (1.5) that cannot hold in general because of Theorem 2.1. We will adapt and modify the arguments in [6] to prove Theorem 2.2. Compared with the perturbed problem in [6, Theorem 5.2] where the Lagrangian coordinates were used, our problem here is coupled to a free interface, rather than the fixed interface $\left\{x_{3}=0\right\}$. As is well-known, the motion of the free surface $\Sigma(t)$ and the domains $\Omega_{ \pm}(t)$ present several mathematical difficulties, so the authors [6] switched the perturbed problem in Eulerian coordinates to a perturbed problem in Lagrangian coordinates, in which the free interface is switched to the fixed interface $\left\{x_{3}=0\right\}$, while the domains of the upper and lower fluids stay fixed in time as $\Omega_{+}=\mathbb{R}^{2} \times(0,1)$ and $\Omega_{-}=\mathbb{R}^{2} \times(-1,0)$, respectively. Thus, the convergence for the jump conditions of the rescaled functions can be easily dealt with at fixed interface in the proof of [6, Theorem 5.2]. To circumvent such difficulties without the aid of the transform of Lagrangian coordinates, similarly to Nespoli and Salvi [12], we transform the perturbed equations $(2.6)_{2}$ to the integral form. Indeed, multiplying $(2.6)_{2}$ by $\phi$, integrating by parts over $\left(0, t_{0}\right) \times \Omega$, and using $(2.7)-(2.9)$ together with the formula of surface integral, we obtain

$$
\begin{aligned}
& \int_{0}^{t_{0}} \int_{\Omega}\left(\varrho \partial_{t} u \cdot \phi+\varrho(\nabla u) u \cdot \phi\right) \mathrm{d} x \mathrm{~d} t+\int_{0}^{t_{0}} \int_{\Omega}\left(\mu\left(\nabla u+\nabla u^{T}\right)-\sigma I\right): \nabla \phi \mathrm{d} x \mathrm{~d} t \\
= & \int_{0}^{t_{0}} \int_{\mathbb{R}^{2}}(g[\varrho] \eta+\kappa H) \phi\left(t, x^{\prime}, \eta\left(t, x^{\prime}\right)\right) \cdot\left(-\partial_{1} \eta,-\partial_{2} \eta, 1\right) \mathrm{d} x^{\prime} \mathrm{d} t,
\end{aligned}
$$

where $\phi:=\left(\phi_{1}, \phi_{2}, \phi_{3}\right) \in\left(\mathcal{D}^{\prime}((0, T) \times \Omega)\right)^{3}$, and

$$
\left(\mu\left(\nabla u+\nabla u^{T}\right)-\sigma I\right): \nabla \phi=\sum_{1 \leq i, j \leq 3}\left(\mu\left(\partial_{j} u_{i}+\partial_{i} u_{j}\right)-\sigma \delta_{i j}\right) \partial_{j} \phi_{i}
$$

In this manner we have transformed the two-fluids flow in a single-fluid flow in a fixed domain, this offers an alternative approach to deal with the jump condition (2.8) at free interface $\Sigma(t)$, instead of using the method of Lagrangian coordinates in $[6,8]$. Consequently, we can avoid the proof of convergence for the jump conditions of the rescaled viscous stress-tensor at the free boundary. This transform will play an important role in the proof of Theorem 2.2 in Section 5 . Moreover, this idea is also applied to the proof of the uniqueness of solutions to the linearized equations (1.5) in Section 4.

We mention that Guo and Tice [8] recently proved the linear global instability for compressible viscous fluids in Lagrangian coordinates, while in the current paper the nonlinear global instability for incompressible viscous fluids in the sense of Definition 2.1 is established in Eulerian coodinates. Finally, we point out that Hwang and Guo [9] constructed an unstable solution to show mathematically the Rayleigh-Taylor instability for two-dimensional, incompressible inviscid flows when the density is continuous. It still needs further study whether we can construct a concrete solution of (2.6)-(2.9) which does not have global stability of order $k$.

\section{Construction of a growing solution to the linearized equations}

\subsection{Growing mode ansatz}

We wish to construct a solution to the linearized equations (1.5) that has a growing $H^{k}$-norm for any $k$. We will construct such solutions via Fourier synthesis by first constructing a growing 
mode for fixed spatial frequency.

To begin, we make a growing mode ansatz, i.e., let us assume that

$$
\eta\left(t, x^{\prime}\right)=\tilde{\eta}\left(x^{\prime}\right) e^{\lambda t}, u(t, x)=v(x) e^{\lambda t}, p(t, x)=q(x) e^{\lambda t}, \quad \text { for some } \lambda>0 .
$$

Substituting this ansatz into (1.5), eliminating $\tilde{\eta}$ by using the first equation, we arrive at the time-invariant system for $v=\left(v_{1}, v_{2}, v_{3}\right)$ and $q$ :

$$
\left\{\begin{array}{l}
\lambda \varrho v+\nabla q=\mu \Delta v \\
\operatorname{div} v=0
\end{array}\right.
$$

with the corresponding jump conditions

$$
\llbracket v \rrbracket=0, \llbracket q I-\mu \nabla\left(v+v^{T}\right) \rrbracket e_{3}=\lambda^{-1}\left(g[\varrho] v_{3}+\kappa \Delta_{x^{\prime}} v_{3}\right) e_{3}
$$

and boundary conditions

$$
v\left(t, x^{\prime},-1\right)=v\left(t, x^{\prime}, 1\right)=0
$$

\subsection{Horizontal Fourier transform}

We take the horizontal Fourier transform of $\left(v_{1}, v_{2}, v_{3}\right)$ in (3.1), which we denote with either $\hat{\imath}$ or $\mathcal{F}$, and fix a spatial frequency $\xi=\left(\xi_{1}, \xi_{2}\right) \in \mathbb{R}^{2}$. Define the new unknowns

$$
\varphi\left(x_{3}\right)=i \hat{v}_{1}\left(\xi, x_{3}\right), \quad \theta\left(x_{3}\right)=i \hat{v}_{2}\left(\xi, x_{3}\right), \quad \psi\left(x_{3}\right)=\hat{v}_{3}\left(\xi, x_{3}\right), \quad \pi\left(x_{3}\right)=\hat{q}\left(\xi, x_{3}\right),
$$

so that

$$
\mathcal{F}(\operatorname{div} w)=\xi_{1} \varphi+\xi_{2} \theta+\psi^{\prime}
$$

where $^{\prime}=d / d x_{3}$. Then, for $\varphi, \theta, \psi$ and $\lambda=\lambda(\xi)$ we arrive at the following system of ODEs.

$$
\left\{\begin{array}{l}
\lambda \varrho \varphi-\xi_{1} \pi+\mu\left(|\xi|^{2} \varphi-\varphi^{\prime \prime}\right)=0 \\
\lambda \varrho \theta-\xi_{2} \pi+\mu\left(|\xi|^{2} \theta-\theta^{\prime \prime}\right)=0 \\
\lambda \varrho \psi+\pi^{\prime}+\mu\left(|\xi|^{2} \psi-\psi^{\prime \prime}\right)=0 \\
\xi_{1} \varphi+\xi_{2} \theta+\psi^{\prime}=0
\end{array}\right.
$$

along with the jump conditions

$$
\left\{\begin{array}{l}
\llbracket \varphi \rrbracket=\llbracket \theta \rrbracket=\llbracket \psi \rrbracket=0, \\
\llbracket \mu\left(\xi_{1} \psi-\varphi^{\prime}\right) \rrbracket=\llbracket \mu\left(\xi_{2} \psi-\theta^{\prime}\right) \rrbracket=0, \\
\llbracket-2 \mu \lambda \psi^{\prime}+\lambda \pi \rrbracket=\left(g[\varrho]-\kappa|\xi|^{2}\right) \psi,
\end{array}\right.
$$

and the boundary conditions

$$
\varphi(-1)=\varphi(1)=\theta(-1)=\theta(1)=\psi(-1)=\psi(1)=0 .
$$

Eliminating $\pi$ from the third equation in (3.2) we obtain the following ODE for $\psi$

$$
-\lambda \rho\left(|\xi|^{2} \psi-\psi^{\prime \prime}\right)=\mu\left(|\xi|^{4} \psi-2|\xi|^{2} \psi^{\prime \prime}+\psi^{\prime \prime \prime \prime}\right)
$$

along with the jump conditions

$$
\begin{aligned}
& \llbracket \psi \rrbracket=\llbracket \psi^{\prime} \rrbracket=0, \\
& \llbracket \mu\left(|\xi|^{2} \psi+\psi^{\prime \prime}\right) \rrbracket=0, \\
& \llbracket \mu \lambda\left(\psi^{\prime \prime \prime}-3|\xi|^{2} \psi^{\prime}\right) \rrbracket=\llbracket \lambda^{2} \varrho \psi^{\prime} \rrbracket+\left(g[\rho]-\kappa|\xi|^{2}\right)|\xi|^{2} \psi,
\end{aligned}
$$

and the boundary conditions

$$
\psi(-1)=\psi(1)=\psi^{\prime}(-1)=\psi^{\prime}(1)=0 .
$$

3.3. Construction of a solution to the fourth order $O D E$

Similarly to $[8,18]$, we can apply the variational methods to construct a solutions of $(3.5)-$ (3.9). For reader's convenience, we sketch the procedure of the construction. 
First, fix a non-zero vector $\xi \in \mathbb{R}^{2}$ and $s>0$. From (3.5)-(3.9) we get a family of the modified problems

$$
-\lambda^{2} \rho\left(|\xi|^{2} \psi-\psi^{\prime \prime}\right)=s \mu\left(|\xi|^{4} \psi-2|\xi|^{2} \psi^{\prime \prime}+\psi^{\prime \prime \prime \prime}\right),
$$

along with the jump conditions

$$
\begin{aligned}
& \llbracket \psi \rrbracket=\llbracket \psi^{\prime} \rrbracket=0, \\
& \llbracket s \mu\left(|\xi|^{2} \psi+\psi^{\prime \prime}\right) \rrbracket=0, \\
& \llbracket s \mu \lambda\left(\psi^{\prime \prime \prime}-3|\xi|^{2} \psi^{\prime}\right) \rrbracket=\llbracket \lambda^{2} \varrho \psi^{\prime} \rrbracket+\left(g[\rho]-\kappa|\xi|^{2}\right)|\xi|^{2} \psi,
\end{aligned}
$$

and the boundary conditions

$$
\psi(-1)=\psi(1)=\psi^{\prime}(-1)=\psi^{\prime}(1)=0 .
$$

We define the energy functional of (3.10) by

$$
E(\psi)=\frac{1}{2} \int_{-1}^{1} s \mu\left(4|\xi|^{2}\left|\psi^{\prime}\right|^{2}+\left.|| \xi\right|^{2} \psi+\left.\psi^{\prime \prime}\right|^{2}\right) \mathrm{d} x_{3}-\frac{1}{2}|\xi|^{2}\left(g[\rho]-\kappa|\xi|^{2}\right)|\psi(0)|^{2}
$$

with a associated admissible set

$$
\mathcal{A}=\left\{\psi \in H_{0}^{2}(-1,1) \mid \int_{-1}^{1} \rho\left(|\xi|^{2}|\psi|^{2}+\left|\psi^{\prime}\right|^{2}\right) \mathrm{d} x_{3}=2\right\}
$$

where $H_{0}^{2}(-1,1)$ is the subset of $H^{2}(-1,1)$ satisfying $(3.14)$. Thus we can find a $-\lambda^{2}$ by minimizing

$$
-\lambda^{2}(|\xi|)=\alpha(|\xi|):=\inf _{\psi \in \mathcal{A}} E(\psi) .
$$

In fact, we can show that a minimizer of (3.17) exists, and that the minimizer satisfies EulerLagrange equations is equivalent to (3.10)-(3.14).

Proposition 3.1. For any fixed $\xi \neq 0, E$ achieves its infinimum on $\mathcal{A}$. In addition, let $\psi$ be a minimizer and $-\lambda^{2}:=E(\psi)$, then the pair $\left(\psi, \lambda^{2}\right)$ satisfies (3.10) along with the jump and boundary conditions (3.11)-(3.14). Moreover, $\psi$ is smooth when restricted to $(-1,0)$ or $(0,1)$.

Proof. We can follow the same proof procedure as in [18, Proposition 3.1] to show Proposition 3.1. Hence, we omit the details of the proof here.

Next, we want to prove that there is a fixed point such that $\lambda=s$. To this end, we shall give some properties of $\alpha(s)$ as a function of $s>0$.

Proposition 3.2. $\alpha(s) \in C_{\mathrm{loc}}^{0,1}(0, \infty)$ is strictly increasing. Moreover,

(1) for any $a, b \in\left(0,|\xi|_{c}\right)$ with $a<b$, there exist constants $c_{1}, c_{2}>0$ depending on $\varrho_{ \pm}, \mu_{ \pm}$, $g, a$ and $b$, such that

$$
\alpha(s) \leq-c_{1}+s c_{2}, \text { for all }|\xi| \in[a, b]
$$

where

$$
|\xi|_{c}:= \begin{cases}\sqrt{g[\varrho] / \kappa}, & \text { if } \kappa>0, \\ +\infty, & \text { if } \kappa=0 .\end{cases}
$$

(2) there exist constants $c_{3}>0$ depending on $\varrho_{ \pm}$and $g, c_{4}>0$ depending additionally on $\mu_{ \pm}$ and $|\xi|$, such that

$$
\alpha(s) \geq-c_{3}|\xi|+s c_{4}
$$


Proof. We refer to [18, Lemma 3.5] and [8, Propostition 3.6] for a proof.

Given $\xi \in \mathbb{R}^{2}$ with $|\xi| \in\left(0,|\xi|_{c}\right)$, by virtue of (3.18), there exists $s_{0}>0$ depending on the quantities $\varrho_{ \pm}, \mu_{ \pm}, g,|\xi|$, so that for $s \leq s_{0}$ it holds that $\alpha(s)<0$. Hence, we can define the open set

$$
\mathcal{S}=\alpha^{-1}(-\infty, 0) \subset(0, \infty)
$$

Note that $\mathcal{S}$ is non-empty and this allows us to define $\lambda(s)=\sqrt{-\alpha(s)}$ for $s \in \mathcal{S}$. Therefore, as a result of Proposition 3.1, we have the following existence result for the modified problem (3.10)-(3.14).

Proposition 3.3. For each $\xi \in \mathbb{R}^{2}$ with $|\xi| \in\left(0,|\xi|_{c}\right)$, and each $s \in \mathcal{S}$ there is a solution $\psi=\psi\left(|\xi|, x_{3}\right)$ with $\lambda=\lambda(|\xi|, s)>0$ to the problem (3.10) with the jump and boundary conditions (3.11)-(3.14). Moreover, $\psi$ is smooth when restricted to $(-1,0)$ or $(0,1)$ with $\psi(|\xi|, 0) \neq 0$.

Finally, we can use Proposition 3.2 to make a fixed-point argument to find $s \in \mathcal{S}$ such that $s=\lambda(|\xi|, s)$ to construct solutions to the original problem (3.5)-(3.9).

Proposition 3.4. Let $\xi \in \mathbb{R}^{2}$ with $|\xi| \in\left(0,|\xi|_{c}\right)$, then there exists a unique $s \in \mathcal{S}$ so that $\lambda(|\xi|, s)=\sqrt{-\alpha(s)}>0$ and $s=\lambda(|\xi|, s)$.

ProOF. We refer to [18, Lemma 3.7] for a proof.

Consequently, in view of Propositions 3.3 and 3.4, we conclude the following existence result concerning the problem of (3.5)-(3.9).

Theorem 3.1. For each $\xi \in \mathbb{R}^{2}$ with $|\xi| \in\left(0,|\xi|_{c}\right)$, there exist $\psi=\psi\left(|\xi|, x_{3}\right)$ and $\lambda(|\xi|)>0$ satisfying (3.5)-(3.9). Moreover, $\psi$ is smooth when restricted to $(-1,0)$ or $(0,1)$ with $\psi(|\xi|, 0) \neq$ 0 .

Next, we show some properties of the solutions established in Theorem 3.1 in terms of $\lambda(|\xi|)$. The first property is given in the following proposition which shows that $\lambda$ is a bounded, continuous function of $|\xi|$.

Proposition 3.5. The function $\lambda:\left(0,|\xi|_{c}\right) \rightarrow(0, \infty)$ is continuous and satisfies

$$
\sup _{0<|\xi|<\infty} \lambda(|\xi|) \leq \frac{g[\varrho]}{4 \mu_{-}}
$$

Moreover,

$$
\lim _{|\xi| \rightarrow 0} \lambda(|\xi|)=0
$$

and if $\kappa>0$, then also

$$
\lim _{|\xi| \rightarrow|\xi|_{c}} \lambda(|\xi|)=0
$$

Proof. The continuity and the limits (3.20), (3.21) follow from the same arguments as in [8, Proposition 3.9] with the help of (3.5), (3.19) and Ehrling-Nirenberg-Gagliardo interpolation inequality. To complete the proof, it suffices to show (3.19). For each $|\xi| \in\left(0,|\xi|_{c}\right)$ there exists a function $\psi_{|\xi|} \in \mathcal{A}$ satisfying (3.5)-(3.9), so that $-\lambda^{2}(|\xi|)=E\left(\psi_{|\xi|}\right)$. By (3.15), we find that

$$
-\lambda^{2}(|\xi|)=\frac{\lambda(|\xi|)}{2} \int_{-1}^{1} \mu\left(4|\xi|^{2}\left|\psi_{|\xi|}^{\prime}\right|^{2}+\left.|| \xi\right|^{2} \psi_{|\xi|}+\left.\psi_{|\xi|}^{\prime \prime}\right|^{2}\right) \mathrm{d} x_{3}-\frac{1}{2}|\xi|^{2}\left(g[\rho]-\kappa|\xi|^{2}\right)\left|\psi_{|\xi|}(0)\right|^{2}
$$


which yields

$$
2 \mu_{-}|\xi|^{2} \lambda(|\xi|) \int_{-1}^{1}\left|\psi_{|\xi|}^{\prime}\right|^{2} \mathrm{~d} x_{3} \leq \frac{1}{2}|\xi|^{2} g[\rho]\left|\psi_{|\xi|}(0)\right|^{2} .
$$

Using the Hölder inequality, we can bound

$$
\left|\psi_{|\xi|}(0)\right|^{2}=\left|\int_{0}^{1} \psi_{|\xi|}^{\prime} \mathrm{d} x_{3}\right|^{2} \leq \int_{0}^{1}\left(\psi_{|\xi|}^{\prime}\right)^{2} \mathrm{~d} x_{3}
$$

Substitution of (3.23) into (3.22) gives then

$$
|\xi|^{2}\left(2 \mu_{-} \lambda(|\xi|)-\frac{1}{2} g[\varrho]\right) \int_{-1}^{1}\left|\psi_{|\xi|}^{\prime}\right|^{2} \mathrm{~d} x_{3} \leq 0 .
$$

Consequently, (3.24) implies (3.19), since $\left\|\psi_{|\xi|}^{\prime}\right\|_{L^{2}(-1,1)}>0$.

\subsection{Construction of a solution to the system (3.2)-(3.4)}

A solution to (3.5)-(3.9) gives rise to a solution of the system (3.2)-(3.4) for the growing mode velocity $v$, as well.

Theorem 3.2. For each $\xi \in \mathbb{R}^{2}$ with $|\xi| \in\left(0,|\xi|_{c}\right)$, there exists a solution $(\tilde{\varphi}, \tilde{\theta}, \tilde{\psi}, \tilde{\pi})=$ $\left(\tilde{\varphi}\left(\xi, x_{3}\right), \tilde{\theta}\left(\xi, x_{3}\right), \tilde{\psi}\left(\xi, x_{3}\right), \tilde{\pi}\left(\xi, x_{3}\right)\right)$ with $\lambda=\lambda(|\xi|)>0$ to (3.2)-(3.4), and the solution is smooth when restricted to $(-1,0)$ or $(0,1)$. Moreover,

$$
\begin{aligned}
& \|\tilde{\varphi}\|_{L^{2}(-1,1)}^{2}+\|\tilde{\theta}\|_{L^{2}(-1,1)}^{2}+\|\tilde{\psi}\|_{L^{2}(-1,1)}^{2}=1, \\
& \left\|\tilde{\psi^{\prime}}\right\|_{L^{2}(-1,1)} \leq|\xi| \sqrt{2 \varrho^{+} \varrho_{-}^{-1}} .
\end{aligned}
$$

Proof. By Theorem 3.1, we first construct a solution $(\psi, \lambda)=\left(\psi\left(|\xi|, x_{3}\right), \lambda(|\xi|)\right)$ satisfying (3.5)-(3.9). Moreover, $\lambda>0$ and $\psi \in \mathcal{A}$ is smooth when restricted to $(-1,0)$ or $(0,1)$. Then, multiplying $(3.2)_{1}$ and $(3.2)_{2}$ by $\xi_{1}$ and $\xi_{2}$ respectively, adding the resulting equations, and utilizing $(3.2)_{4}$, we find that $\pi$ can be expressed by $\psi$, i.e.,

$$
\pi=\pi\left(|\xi|, x_{3}\right)=\left[\mu \psi^{\prime \prime \prime}-\left(\lambda \varrho+\mu|\xi|^{2}\right) \psi^{\prime}\right]|\xi|^{-2}
$$

Notice that $(3.2)_{1}$ can be rewritten as

$$
\varphi^{\prime \prime}-\left(\lambda \varrho+\mu|\xi|^{2}\right) \varphi / \mu=-\xi_{1} \pi / \mu
$$

with jump and boundary conditions

$$
\llbracket \varphi \rrbracket=0, \llbracket \mu\left(\xi_{1} \psi-\varphi^{\prime}\right) \rrbracket=0, \varphi(-1)=\varphi(1)=0 .
$$

Hence, we can easily construct a unique solution

$$
\varphi=\left(\xi, x_{3}\right)=\left\{\begin{array}{l}
\xi_{1}\left(c_{1} e^{a_{+} x_{3}}+c_{2} e^{-a_{+} x_{3}}-f_{+}\left(x_{3}\right)\right), \text { on }(0,1), \\
\xi_{1}\left(c_{3} e^{a_{-} x_{3}}+c_{4} e^{-a_{-} x_{3}}-f_{-}\left(x_{3}\right)\right), \text { on }(-1,0)
\end{array}\right.
$$

to the equation (3.28) with jump and boundary conditions (3.29), where

$$
\begin{aligned}
& a_{ \pm}=\sqrt{|\xi|^{2}+\lambda \varrho / \mu_{ \pm}} \\
& f_{ \pm}\left(x_{3}\right)=\frac{1}{2 a_{ \pm} \mu_{ \pm}} \int_{0}^{x_{3}} \pi\left(e^{a_{ \pm}\left(x_{3}-y\right)}-e^{a_{ \pm}\left(y-x_{3}\right)}\right) \mathrm{d} y
\end{aligned}
$$


and

$$
\left[\begin{array}{c}
c_{1} \\
c_{2} \\
c_{3} \\
c_{4}
\end{array}\right]=\left[\begin{array}{c}
0 \\
\left(\mu_{+}-\mu_{-}\right) \psi(0) \\
f(1) \\
f(-1)
\end{array}\right]\left[\begin{array}{cccc}
1 & 1 & -1 & -1 \\
\mu_{+} a_{+} & -\mu_{+} a_{+} & -\mu_{-} a_{-} \mu_{-} a_{-} \\
e^{a_{+}} & e^{-a_{+}} & 0 & 0 \\
0 & 0 & e^{-a_{-}} & e^{a_{-}}
\end{array}\right]^{-1}
$$

Similarly to $(3.30)$,

$$
\theta:=\theta\left(\xi, x_{3}\right)=\left\{\begin{array}{l}
\xi_{2}\left(c_{1} e^{a_{+} x_{3}}+c_{2} e^{-a_{+} x_{3}}-f_{+}\left(x_{3}\right)\right), \text { on }(0,1), \\
\xi_{2}\left(c_{3} e^{a_{-} x_{3}}+c_{4} e^{-a_{-} x_{3}}-f_{-}\left(x_{3}\right)\right), \text { on }(-1,0)
\end{array}\right.
$$

is a unique solution of $(3.2)_{2}$ with jump and boundary conditions:

$$
\llbracket \theta \rrbracket=0, \llbracket \mu\left(\xi_{2} \psi-\theta^{\prime}\right) \rrbracket=0, \theta(-1)=\theta(1)=0 .
$$

Consequently, $(\varphi, \theta, \psi, \pi)$ is a solution to the system (3.2)-(3.4). Now, we define

$$
\begin{aligned}
(\tilde{\varphi}, \tilde{\theta}, \tilde{\psi}, \tilde{\pi}) & :=\left(\tilde{\varphi}\left(\xi, x_{3}\right), \tilde{\theta}\left(\xi, x_{3}\right), \tilde{\psi}\left(\xi, x_{3}\right), \tilde{\pi}\left(\xi, x_{3}\right)\right) \\
& :=(\varphi, \theta, \psi, \pi) /\left(\|\varphi\|_{L^{2}(-1,1)}^{2}+\|\theta\|_{L^{2}(-1,1)}^{2}+\|\psi\|_{L^{2}(-1,1)}^{2}\right) .
\end{aligned}
$$

Thus, $(\tilde{\varphi}, \tilde{\theta}, \tilde{\psi}, \tilde{\pi})$ is still a solution to the system $(3.2)-(3.4)$, and moreover, $(\tilde{\varphi}, \tilde{\theta}, \tilde{\psi}, \tilde{\pi})$ satisfies (3.25).

Finally, making use of $(3.2)_{4}$ and (3.16), we conclude that

$$
\begin{aligned}
\frac{1}{\varrho^{+}|\xi|^{2}} & =\frac{1}{2 \varrho^{+}|\xi|^{2}} \int_{-1}^{1} \varrho\left(|\xi|^{2}|\psi|^{2}+\left|\psi^{\prime}\right|^{2}\right) \mathrm{d} x_{3} \\
& \leq \int_{-1}^{1}\left(|\varphi|^{2}+|\theta|^{2}+|\psi|^{2}\right) \mathrm{d} x_{3} \\
& \leq\|\varphi\|_{L^{2}(-1,1)}^{2}+\|\theta\|_{L^{2}(-1,1)}^{2}+\|\psi\|_{L^{2}(-1,1)}^{2}
\end{aligned}
$$

and

$$
\int_{-1}^{1}\left|\psi^{\prime}\right|^{2} \mathrm{~d} x_{3} \leq 2 / \varrho_{-} .
$$

The above two inequalities imply (3.26) immediately.

Remark 3.1. For each $x_{3}$, it is easy to see that the solution $(\tilde{\varphi}(\xi, \cdot), \tilde{\theta}(\xi, \cdot), \tilde{\psi}(\xi, \cdot), \tilde{\pi}(\xi, \cdot), \lambda(|\xi|))$ constructed in Theorem 3.2 has the following properties:

(1) $\lambda(|\xi|), \tilde{\psi}(\xi, \cdot)$ and $\tilde{\pi}(\xi, \cdot)$ are even on $\xi_{1}$ or $\xi_{2}$, when the another variable is fixed;

(2) $\tilde{\varphi}(\xi, \cdot)$ is odd on $\xi_{1}$, but even on $\xi_{2}$, when the another variable is fixed;

(3) $\tilde{\theta}(\xi, \cdot)$ is even on $\xi_{1}$, but odd on $\xi_{2}$, when the another variable is fixed.

To directly estimate the $H^{k}$ norm of the solution $\psi$ from (3.5), without use of (3.15) and the continuity of $\lambda$, we shall apply the following Ehrling-Nirenberg-Gagliardo interpolation inequality, the proof of which can be found in [1, Chapter 5].

Lemma 3.1. Let $\Omega$ be a domain in $\mathbb{R}^{n}$ satisfying the cone condition. For each $\varepsilon_{0}>0$ there is a constant $K$ depending on $n, m, p$ and $\varepsilon_{0}$, such that if $0<\varepsilon \leq \varepsilon_{0}, 0 \leq j \leq m$, and $u \in W^{m, p}(\Omega)$, then

$$
\sum_{|\alpha|=j} \int_{\Omega}\left|D^{\alpha} u(x)\right|^{p} \mathrm{~d} x \leq K\left(\varepsilon \sum_{|\alpha|=m} \int_{\Omega}\left|D^{\alpha} u(x)\right|^{p} \mathrm{~d} x+\varepsilon^{-j /(m-j)} \int_{\Omega}|u|^{p} \mathrm{~d} x\right) .
$$


The next lemma provides an estimate for the $H^{k}$ norm of the solution $(\tilde{\varphi}, \tilde{\theta}, \tilde{\psi}, \tilde{\pi})$ with $\xi$ varying, which will be useful in the next section when such a solution is integrated in a Fourier synthesis. To emphasize the dependence on $\xi$, we write these solutions as $\left(\tilde{\varphi}(\xi)=\tilde{\varphi}\left(\xi, x_{3}\right), \tilde{\theta}(\xi)=\right.$ $\left.\tilde{\theta}\left(\xi, x_{3}\right), \tilde{\psi}(\xi)=\tilde{\psi}\left(\xi, x_{3}\right), \tilde{\pi}(\xi)=\tilde{\pi}\left(\xi, x_{3}\right)\right)$.

Lemma 3.2. Let $\xi \in \mathbb{R}^{2}$ with $0<R_{1}<|\xi|<|\xi|_{c}, \varphi(\xi):=\tilde{\varphi}(\xi), \theta(\xi):=\tilde{\theta}(\xi), \psi(\xi):=\tilde{\psi}(\xi)$, $\pi(\xi):=\tilde{\pi}(\xi)$ and $\lambda(|\xi|)$ be constructed as in Theorem 3.2, then for any $k \geq 0$ there exit positive constants $a_{k}, b_{k}$ and $c_{k}$ depending on $R_{1}, \varrho_{ \pm}, \mu_{ \pm}$and $g$, so that

$$
\begin{aligned}
& \|\varphi(\xi)\|_{H^{k}(-1,0)}+\|\varphi(\xi)\|_{H^{k}(0,1)}+\|\theta(\xi)\|_{H^{k}(-1,0)}+\|\theta(\xi)\|_{H^{k}(0,1)} \leq a_{k} \sum_{j=0}^{k+1}|\xi|^{j} \\
& \|\psi(\xi)\|_{H^{k}(-1,0)}+\|\psi(\xi)\|_{H^{k}(0,1)} \leq b_{k} \sum_{j=0}^{k}|\xi|^{j} \\
& \|\pi(\xi)\|_{H^{k}(-1,0)}+\|\pi(\xi)\|_{H^{k}(0,1)} \leq c_{k} \sum_{j=0}^{k+1}|\xi|^{j} .
\end{aligned}
$$

Moreover,

$$
\|\varphi\|_{L^{2}(-1,1)}^{2}+\|\theta\|_{L^{2}(-1,1)}^{2}+\|\psi\|_{L^{2}(-1,1)}^{2}=1 .
$$

Proof. Throughout this proof, we denote by $\tilde{c}_{1}, \cdots, \tilde{c}_{9}$ generic positive constants which may depend on $R_{1}, \varrho_{ \pm}, \mu_{ \pm}$and $g$, but not on $|\xi|$.

(i) First, (3.34) follows from (3.25) immediately. We now write (3.5) as

$$
\psi^{\prime \prime \prime \prime}(\xi)=\left[\left(\lambda \varrho+2 \mu|\xi|^{2}\right) \psi^{\prime \prime}(\xi)-\left(\lambda \varrho|\xi|^{2}+\mu|\xi|^{4}\right) \psi(\xi)\right] / \mu .
$$

If we make use of (3.19), $|\xi|>R_{1}$ and Lemma 3.1, then we see that there exists a couple $\left(\tilde{c}_{1}, \tilde{c}_{2}\right)$, such that

$$
\begin{aligned}
\left\|\psi^{\prime \prime \prime \prime}(\xi)\right\|_{L^{2}\left(I_{ \pm}\right)} \leq & \tilde{c}_{1}\left[\left(|\xi|^{2}+|\xi|^{4}\right)\|\psi(\xi)\|_{L^{2}\left(I_{ \pm}\right)}+\left(1+|\xi|^{2}\right)\left\|\psi^{\prime \prime}(\xi)\right\|_{L^{2}\left(I_{ \pm}\right)}\right] \\
\leq & \left(\tilde{c}_{2}+1\right)\left[\left(\varepsilon^{-1 / 2}+\varepsilon^{-1 / 2}|\xi|^{4}+|\xi|^{2}+|\xi|^{4}\right)\|\psi(\xi)\|_{L^{2}(I \pm)}\right. \\
& \left.+\sqrt{\varepsilon}\left\|\psi^{\prime \prime \prime \prime}(\xi)\right\|_{L^{2}\left(I_{ \pm}\right)}\right] \quad \text { for any } \varepsilon \in(0,1),
\end{aligned}
$$

respectively, where $I_{+}=(0,1)$ and $I_{-}=(-1,0)$. Choosing $\sqrt{\varepsilon}=1 /\left\{2\left(\tilde{c}_{2}+1\right)\right\}$ in $(3.36)$ and using (3.34), we arrive at

$$
\left\|\psi^{\prime \prime \prime \prime}(\xi)\right\|_{L^{2}\left(I_{ \pm}\right)} \leq \tilde{c}_{3}\left(1+|\xi|^{2}+|\xi|^{4}\right) \text { for some } \tilde{c}_{3}>0,
$$

whence,

$$
\left\|\psi^{\prime \prime \prime \prime}(\xi)\right\|_{L^{2}(-1,1)} \leq \tilde{c}_{4}\left(1+|\xi|^{2}+|\xi|^{4}\right)
$$

Writing (3.35) as

$$
\psi^{\prime \prime}(\xi)=\frac{\mu \psi^{\prime \prime \prime \prime}(\xi)+\left(\lambda \varrho|\xi|^{2}+\mu|\xi|^{4}\right) \psi(\xi)}{\left(\lambda \varrho+2 \mu|\xi|^{2}\right)},
$$

we utilize (3.37) and (3.34) to get

$$
\left\|\psi^{\prime \prime}(\xi)\right\|_{L^{2}(-1,1)} \leq \tilde{c}_{5}\left(1+|\xi|^{2}\right) .
$$

Differentiating (3.35) with respect to $x_{3}$, we see that

$$
\psi^{(5)}(\xi)=\left[\left(\lambda \varrho+2 \mu|\xi|^{2}\right) \psi^{\prime \prime \prime}(\xi)-\left(\lambda \varrho|\xi|^{2}+\mu|\xi|^{4}\right) \psi^{\prime}(\xi)\right] / \mu .
$$


Similarly to (3.37)-(3.38), we obtain, by Lemma 3.1, (3.19), (3.26) and (3.34), that

$$
\left\|\psi^{(5)}(\xi)\right\|_{L^{2}(-1,1)} \leq \tilde{c}_{6}\left(1+|\xi|^{2}+|\xi|^{4}+|\xi|^{5}\right) .
$$

and

$$
\left\|\psi^{\prime \prime \prime}(\xi)\right\|_{L^{2}(-1,1)} \leq \tilde{c}_{7}\left(1+|\xi|^{2}+|\xi|^{3}\right) .
$$

Summarizing the estimates (3.34), (3.26) and (3.37)-(3.40), we conclude that, for each nonnegative integer $k \in[0,5]$, there exists a constant $\tilde{b}_{k}>0$ depending on $R_{1}, \varrho_{ \pm}, \mu_{ \pm}$and $g$, such that

$$
\left\|\psi^{(k)}(\xi)\right\|_{L^{2}(-1,1)} \leq \tilde{b}_{k} \sum_{j=0}^{k}|\xi|^{j}
$$

Differentiating (3.35) with respect to $x_{3}$ and using (3.41), we find, by induction on $k$, that (3.41) holds for any $k \geq 0$. This gives (3.32).

(ii) Recalling the expression (3.27) of $\pi$ and the fact that $|\xi|>R_{1}$, we employ (3.32) to deduce that for any $k \geq 0$,

$$
\begin{gathered}
\left\|\pi^{(k)}(\xi)\right\|_{L^{2}(-1,1)} \leq \frac{\mu^{+}}{|\xi|^{2}}\left\|\psi^{(k+3)}(\xi)\right\|_{L^{2}(-1,1)}+\left(\frac{g[\varrho] \varrho^{+}}{4 \mu_{-}|\xi|^{2}}+\mu^{+}\right)\left\|\psi^{(k+1)}(\xi)\right\|_{L^{2}(-1,1)} \\
\leq \max \left\{\frac{\mu^{+} \tilde{b}_{k+3}}{R_{1}^{2}}+\frac{\mu^{+} \tilde{b}_{k+3}}{R_{1}}+\mu^{+} \tilde{b}_{k+3}+\left(\frac{g[\varrho] \varrho^{+}}{4 \mu_{-} R_{1}^{2}}+\mu^{+}\right) \tilde{b}_{k+1},\right. \\
\left.\mu^{+} \tilde{b}_{k+3}+\left(\frac{g[\varrho] \varrho^{+}}{4 \mu_{-} R_{1}^{2}}+\mu^{+}\right) \tilde{b}_{k+1}\right\} \sum_{j=0}^{k+1}|\xi|^{j}
\end{gathered}
$$

which implies (3.33).

(iii) From $(3.19),(3.28),(3.33)$ and $(3.34)$ we get

$$
\begin{aligned}
\left\|\varphi^{\prime \prime}(\xi)\right\|_{L^{2}(-1,1)} & \leq \frac{|\xi|}{\mu_{-}}\|\pi(\xi)\|_{L^{2}(-1,1)}+\left(\frac{g[\varrho] \varrho^{+}}{4 \mu_{-}^{2}}+|\xi|^{2}\right)\|\varphi(\xi)\|_{L^{2}(-1,1)} \\
& \leq \tilde{c}_{8}\left(1+|\xi|+|\xi|^{2}\right) .
\end{aligned}
$$

Applying (3.42), (3.34) and Lemma 3.1, we obtain

$$
\left\|\varphi^{\prime}(\xi)\right\|_{L^{2}(-1,0)}+\left\|\varphi^{\prime}(\xi)\right\|_{L^{2}(0,1)} \leq \tilde{c}_{9}\left(1+|\xi|+|\xi|^{2}\right) .
$$

Combining (3.34) with (3.42) and (3.43), we conclude that, for each nonnegative integer $k \in[0,2]$, there exists a constant $\tilde{a}_{k}>0$ depending on $R_{1}, \varrho_{ \pm}, \mu_{ \pm}$and $g$, so that

$$
\left\|\varphi^{(k)}(\xi)\right\|_{L^{2}(-1,1)} \leq \tilde{a}_{k} \sum_{j=0}^{k+1}|\xi|^{j} .
$$

Thus, by virtue of (3.28), (3.44) and induction on $k$, (3.44) holds for any $k \geq 0$. Following the same procedure as used in estimating $\varphi$, we infer that for each $k \geq 0$,

$$
\left\|\theta^{(k)}(\xi)\right\|_{L^{2}(-1,1)} \leq \tilde{d}_{k} \sum_{j=0}^{k+1}|\xi|^{j}
$$

for some constant $\tilde{d}_{k}$ depending on $R_{1}, \varrho_{ \pm}, \mu_{ \pm}$and $g$. Adding (3.45) to (3.44), we arrive at

$$
\left\|\varphi^{(k)}(\xi)\right\|_{L^{2}(-1,1)}+\left\|\theta^{(k)}(\xi)\right\|_{L^{2}(-1,1)} \leq\left(\tilde{a}_{k}+\tilde{d}_{k}\right) \sum_{j=0}^{k+1}|\xi|^{j} \text { for any } k \geq 0,
$$

which yields (3.31). This completes the proof. 


\subsection{Fourier synthesis}

In this section we will use the Fourier synthesis to build growing solutions to (1.5) out of the solutions constructed in the previous section (Theorem 3.2) for fixed spatial frequency $\xi \in \mathbb{R}^{2}$ with $|\xi| \in\left(0,|\xi|_{c}\right)$. The constructed solutions will grow in-time in the piecewise Sobolev space of order $k, H^{k}\left(\Omega_{ \pm}\right)$, defined by (2.1).

Theorem 3.3. Let $0<R_{1}<R_{2}<|\xi|_{c}$ and $f \in C_{0}^{\infty}\left(R_{1}, R_{2}\right)$ be a real-valued function. For $\xi \in \mathbb{R}^{2}$ with $|\xi| \in\left(0,|\xi|_{c}\right)$, define

$$
v\left(\xi, x_{3}\right)=-i \varphi\left(\xi, x_{3}\right) e_{1}-i \theta\left(\xi, x_{3}\right) e_{2}+\psi\left(\xi, x_{3}\right) e_{3},
$$

where $(\varphi, \theta, \psi, \pi)\left(\xi, x_{3}\right):=(\tilde{\varphi}, \tilde{\theta}, \tilde{\psi}, \tilde{\pi})\left(\xi, x_{3}\right)$ with $\lambda(|\xi|)>0$ is the solution given by Theorem 3.2. Denote

$$
\begin{aligned}
& \eta\left(t, x^{\prime}\right)=\frac{1}{4 \pi^{2}} \int_{\mathbb{R}^{2}} f(|\xi|) v_{3}(\xi, 0) e^{\lambda(|\xi|) t} e^{i x^{\prime} \xi} \mathrm{d} \xi, \\
& u(t, x)=\frac{1}{4 \pi^{2}} \int_{\mathbb{R}^{2}} \lambda(\xi) f(|\xi|) v\left(\xi, x_{3}\right) e^{\lambda(|\xi|) t} e^{i x^{\prime} \xi} \mathrm{d} \xi \\
& p(t, x)=\frac{1}{4 \pi^{2}} \int_{\mathbb{R}^{2}} \lambda(\xi) f(|\xi|) \pi\left(\xi, x_{3}\right) e^{\lambda(|\xi|) t} e^{i x^{\prime} \xi} \mathrm{d} \xi,
\end{aligned}
$$

Then, $(\eta, u, p)$ is a real-valued solution to the linearized problem (1.5) along with the corresponding jump and boundary conditions. For every $k \in \mathbb{N}$, we have the estimate

$$
\begin{aligned}
& \|\eta(0)\|_{H^{k}\left(\mathbb{R}^{2}\right)}+\|u(0)\|_{H^{k}\left(\Omega_{ \pm}\right)}+\|p(0)\|_{H^{k}\left(\Omega_{ \pm}\right)} \\
& \quad \leq \tilde{c}_{k}\left(\int_{\mathbb{R}^{2}}\left(1+|\xi|^{2}\right)^{k+2}|f(|\xi|)|^{2} \mathrm{~d} \xi\right)^{1 / 2}<\infty,
\end{aligned}
$$

where $\tilde{c}_{k}>0$ is a constant depending on the parameters $\varrho_{ \pm}, R_{1}$ and $g$. Moreover, for every $t>0$ we have $\eta(t) \in H^{k}\left(\mathbb{R}^{2}\right), u(t), p(t) \in H^{k}\left(\Omega_{ \pm}\right)$, and

$$
\begin{aligned}
& e^{t \lambda_{0}(f)}\|\eta(0)\|_{H^{k}\left(\mathbb{R}^{2}\right)} \leq\|\eta(t)\|_{H^{k}\left(\mathbb{R}^{2}\right)} \leq e^{t \Lambda}\|\eta(0)\|_{H^{k}\left(\mathbb{R}^{2}\right)}, \\
& e^{t \lambda_{0}(f)}\|u(0)\|_{H^{k}\left(\Omega_{ \pm}\right)} \leq\|u(t)\|_{H^{k}\left(\Omega_{ \pm}\right)} \leq e^{t \Lambda}\|u(0)\|_{H^{k}\left(\Omega_{ \pm}\right)}, \\
& e^{t \lambda_{0}(f)}\|p(0)\|_{H^{k}\left(\Omega_{ \pm}\right)} \leq\|p(t)\|_{H^{k}\left(\Omega_{ \pm}\right)} \leq e^{t \Lambda}\|p(0)\|_{H^{k}\left(\Omega_{ \pm}\right)},
\end{aligned}
$$

where

$$
\lambda_{0}(f)=\inf _{|\xi| \in \operatorname{supp}(f)} \lambda(|\xi|)>0
$$

and

$$
\Lambda=\sup _{0<|\xi|<|\xi|_{c}} \lambda(|\xi|)<\frac{g[\varrho]}{4 \mu_{-}} .
$$

Proof. By virtue of Proposition 3.5, (3.53) and (3.54) hold. For each fixed $\xi \in \mathbb{R}^{2}$,

$$
\begin{aligned}
& \eta\left(t, x^{\prime}\right)=f(|\xi|) v_{3}(\xi, 0) e^{\lambda(|\xi|) t} e^{i x^{\prime} \xi} \\
& u(t, x)=\lambda(|\xi|) f(|\xi|) v\left(\xi, x_{3}\right) e^{\lambda(|\xi|) t} e^{i x^{\prime} \xi} \\
& p(t, x)=\lambda(|\xi|) f(|\xi|) \pi\left(\xi, x_{3}\right) e^{\lambda(|\xi|) t} e^{i x^{\prime} \xi}
\end{aligned}
$$

give a solution to (1.5). Since $f \in C_{0}^{\infty}\left(R_{1}, R_{2}\right)$, Lemma 3.2 implies that

$$
\sup _{\xi \in \operatorname{supp}(f)}\left\|\partial_{3}^{k} v(\xi, \cdot)\right\|_{L^{\infty}}<\infty \quad \text { for all } k \in \mathbb{N} .
$$


Also, $\lambda(\xi) \leq \Lambda$. These bounds show that the Fourier synthesis of the solution given by (3.46)(3.48) is also a solution of (1.5). Because $f$ is real-valued and radial, combined with Remark 3.1, we can easily verify that the Fourier synthesis is real-valued.

The estimate (3.49) follows from Lemma 3.2 with arbitrary $k \geq 0$ and the fact that $f$ is compactly supported. Finally, we can use (3.53), (3.54) and (3.46)-(3.48) to obtain the estimates (3.50)-(3.52).

\section{Global instability for the linearized problem}

\subsection{Uniqueness of linearized equations}

In this subsection, we will show the uniqueness of solutions to the linearized problem, which will be applied to prove Theorem 2.2 in Section 5. For this purpose, we need a generalized formula of integrating by parts (or Gauss-Green's formula). Let us first recall the boundary trace theorem (see Theorem 5.36 in [1, Chaperter 5]).

Lemma 4.1. Let $U$ be a domain in $\mathbb{R}^{n}$ satisfying the uniform $C^{m}$-regularity condition, and assume that there exists a simple $(m, p)$-extension operator $E$ for $U$. Also assume that $m p<n$ and $p \leq q \leq p^{*}=(n-1) p /(n-m p)$. Then, there exists a bounded linear operator

$$
\gamma^{U}: W^{m, p}(U) \rightarrow L^{q}(\partial U)
$$

such that

$$
\gamma^{U}(u)=u \text { on } \partial U
$$

for all $u \in W^{m, p}(U) \cap C(\bar{U})$.

The function $\gamma^{U}(u) \in L^{q}(\partial U)$ is called the trace of the function of $u \in W^{1, p}(U)$ on the boundary $\partial U$. By the Stein extension theorem (see Theorem 5.24 in [1, Chaperter 5]) and the definition of the uniform $C^{m}$-regularity condition (see Definition 4.10 in [1, Chaperter 5]), it is easy to verify that $\Omega, \Omega_{+}$and $\Omega_{-}$have different simple $(m, p)$-extension operators. Keeping these facts in mind, we can start to show the following formula of integrating by parts. For convenience in the subsequent analysis, we will use the natations $\gamma_{+}(f):=\gamma^{\Omega_{+}}\left(f_{+}\right)$and $\gamma_{-}(f):=\gamma^{\Omega_{+}}\left(f_{-}\right)$.

Lemma 4.2. For all $u \in H_{0}^{1}(\Omega)$ and $w \in H^{1}\left(\Omega_{ \pm}\right)$, we have

$$
\int_{\Omega} \partial_{i} w u \mathrm{~d} x=-\int_{\Omega} w \partial_{i} u \mathrm{~d} x+\int_{\mathbb{R}^{2}}\left(\gamma_{+}(w)-\gamma_{-}(w)\right) \gamma_{+}(u) \alpha_{i} \mathrm{~d} x
$$

for $i=1,2,3$, where $\alpha_{1}=\alpha_{2}=0$ and $\alpha_{3}=-1$.

Proof. Temporarily suppose $\bar{u} \in C_{0}^{1}(\Omega), \bar{w}_{+} \in C^{1}\left(\bar{\Omega}_{+}\right)$and $\bar{w}_{-} \in C^{1}\left(\bar{\Omega}_{-}\right)$. By the Gauss-Green theorem, we have

$$
\int_{\Omega} \partial_{i} \bar{w} \bar{u} \mathrm{~d} x=-\int_{\Omega} \bar{w} \partial_{i} \bar{u} \mathrm{~d} x+\int_{\mathbb{R}^{2}}\left(\left(\bar{w}_{+}-\bar{w}_{-}\right) \bar{u}\right)\left(x^{\prime}, 0\right) \alpha_{i} \mathrm{~d} x
$$

Using Lemma 4.1, one has

$$
\begin{aligned}
\left\|\left(\bar{u}-\gamma_{+}(u)\right)\left(x^{\prime}, 0\right)\right\|_{L^{2}\left(\mathbb{R}^{2}\right)} & \leq\left\|\bar{u}-\gamma_{+}(u)\right\|_{L^{2}\left(\partial \Omega_{+}\right)} \\
& =\left\|\gamma_{+}(\bar{u}-u)\right\|_{L^{2}\left(\partial \Omega_{+}\right)} \leq c\|\bar{u}-u\|_{H^{1}\left(\Omega_{+}\right)} \\
& \leq c\|\bar{u}-u\|_{H_{0}^{1}(\Omega)}
\end{aligned}
$$


and

$$
\left\|\left(\bar{w}_{+}-\gamma_{+}\left(w_{+}\right)\right)\left(x^{\prime}, 0\right)\right\|_{L^{2}\left(\mathbb{R}^{2}\right)} \leq c\left\|\bar{w}_{+}-w_{+}\right\|_{H^{1}\left(\Omega_{+}\right)}
$$

for some constant $c>0$. By the Hölder inequality, the above two estimates imply that

$$
\begin{aligned}
& \left\|\left(\bar{w}_{+} \bar{u}-\gamma_{+}(w) \gamma_{+}(u)\right)\left(x^{\prime}, 0\right)\right\|_{L^{1}\left(\mathbb{R}^{2}\right)} \\
& \leq\left\|\left(\bar{u}\left(\bar{w}_{+}-\gamma_{+}(w)\right)\right)\left(x^{\prime}, 0\right)\right\|_{L^{1}\left(\mathbb{R}^{2}\right)}+\left\|\left(\gamma_{+}(w)\left(\bar{u}-\gamma_{+}(u)\right)\right)\left(x^{\prime}, 0\right)\right\|_{L^{1}\left(\mathbb{R}^{2}\right)} \\
& \leq\left\|\bar{u}\left(x^{\prime}, 0\right)\right\|_{L^{2}\left(\mathbb{R}^{2}\right)}\left\|\left(\bar{w}_{+}-\gamma_{+}(w)\right)\left(x^{\prime}, 0\right)\right\|_{L^{2}\left(\mathbb{R}^{2}\right)} \\
& \quad+\left\|\gamma_{+}(w)\left(x^{\prime}, 0\right)\right\|_{L^{2}\left(\mathbb{R}^{2}\right)}\left\|\left(\bar{u}-\gamma_{+}(u)\right)\left(x^{\prime}, 0\right)\right\|_{L^{2}\left(\mathbb{R}^{2}\right)} \\
& \leq c^{2}\|\bar{u}\|_{H_{0}^{1}(\Omega)}\left\|\bar{w}_{+}-w_{+}\right\|_{H^{1}\left(\Omega_{+}\right)}+c^{2}\left\|w_{+}\right\|_{H^{1}\left(\Omega_{+}\right)}\|\bar{u}-u\|_{H_{0}^{1}(\Omega)} .
\end{aligned}
$$

Similarly to (4.3), one gets

$$
\begin{aligned}
& \left\|\left(\bar{w}_{-} \bar{u}-\gamma_{-}(w) \gamma_{+}(u)\right)\left(x^{\prime}, 0\right)\right\|_{L^{1}\left(\mathbb{R}^{2}\right)} \\
& \quad \leq c^{2}\left(\|\bar{u}\|_{H_{0}^{1}(\Omega)}\left\|\bar{w}_{-}-w_{-}\right\|_{H^{1}\left(\Omega_{-}\right)}+\left\|w_{-}\right\|_{H^{1}\left(\Omega_{-}\right)}\|\bar{u}-u\|_{H_{0}^{1}(\Omega)}\right) .
\end{aligned}
$$

In addition, if $\bar{u}_{m} \rightarrow u$ strongly in $H_{0}^{1}(\Omega)$, then there exists $m_{0}>0$ such that

$$
\left\|\bar{u}_{m}\right\|_{H_{0}^{1}(\Omega)} \leq\|u\|_{H_{0}^{1}(\Omega)}+1 \quad \text { for any } m \geq m_{0} .
$$

Note that since $C_{0}(\Omega)$ is dense in $H_{0}^{1}(\Omega)$, and $C_{0}\left(\mathbb{R}^{3}\right)$ is dense in $H^{1}\left(\Omega_{+}\right)$or $H^{1}\left(\Omega_{-}\right)$, thus (4.1) follows from (4.2)-(4.5), using a standard density argument.

Definition 4.1. Given $t_{0}>0$ and the initial data $\left(\eta_{0}, u_{0}\right)$ to the linearized problem (1.5)-(1.7), a triple $(\eta, u, p)$ is called a strong solution of (1.5)-(1.7), if

(1) $\eta \in C^{0}\left(\left[0, t_{0}\right], L_{\mathrm{loc}}^{2}\left(\mathbb{R}^{2}\right)\right), u \in C^{0}\left(\left[0, t_{0}\right],\left(L^{2}(\Omega)\right)^{3}\right), \eta(0)=\eta_{0}, u(0)=u_{0}$ and

$$
\operatorname{ess} \sup _{0<t<t_{0}}\left(\|u(t)\|_{H^{3}\left(\Omega_{ \pm}\right)}+\|\eta(t)\|_{H^{2}\left(\mathbb{R}^{2}\right)}+\|u(t)\|_{H_{0}^{1}(\Omega)}+\|p(t)\|_{H^{1}\left(\Omega_{ \pm}\right)}\right)<\infty .
$$

(2) the equations

$$
\begin{aligned}
& \varrho \partial_{t} u+\nabla p=\mu \Delta u, \\
& \operatorname{div} u=0
\end{aligned}
$$

hold a.e. in $\left(0, t_{0}\right) \times\left(\Omega \backslash\left\{x_{3}=0\right\}\right)$.

(3) For a.e. $t \in\left(0, t_{0}\right)$,

$$
\begin{aligned}
& \partial_{t} \eta=u_{3}, \\
& \left(\left(\lambda_{+}(p) I-\mu_{+}\left(\nabla u_{+}+\nabla u_{+}^{T}\right)\right)-\left(\lambda_{-}(p) I-\mu_{-}\left(\nabla u_{-}+\nabla u_{-}^{T}\right)\right)\right) \cdot e_{3} \\
& \quad=\left(g[\varrho] \eta+\kappa \Delta_{x^{\prime}} \eta\right) e_{3}
\end{aligned}
$$

holds a.e. in $\mathbb{R}^{2} \times\left\{x_{3}=0\right\}$, where $u_{3}$ is the third component of $u$.

Remark 4.1. Since $u(t) \in H_{0}^{1}(\Omega) \cap H^{3}\left(\Omega_{ \pm}\right)$for each $t \geq 0$, we can make use of the embedding theorem and (4.8) to obtain

$$
u(t) \in C^{0}(\bar{\Omega}), u_{+}(t) \in C^{1}\left(\bar{\Omega}_{+}\right), u_{-}(t) \in C^{1}\left(\bar{\Omega}_{-}\right),
$$

and

$$
\begin{aligned}
& u(t) \equiv 0 \text { on } \partial \Omega, \\
& \nabla_{x^{\prime}} u_{+} \equiv \nabla_{x^{\prime}} u_{-} \text {on } \mathbb{R}^{2}, \\
& \operatorname{div} u(t) \equiv 0 \text { in } \bar{\Omega} \quad \text { for a.e. } t \geq 0 .
\end{aligned}
$$


Thus, in view of (4.13), we define for the sake of simplicity that

$$
\nabla_{x^{\prime}} u:=\nabla_{x^{\prime}} u_{+}=\nabla_{x^{\prime}} u_{-} \text {on } \mathbb{R}^{2} \times\{0\} .
$$

Moreover, by virtue of Lemma 4.1, there is a constant $c$ such that

$$
\left\|u\left(t, x^{\prime}, 0\right)\right\|_{H^{1}\left(\mathbb{R}^{2}\right)} \leq c\|u(t)\|_{H^{2}\left(\Omega_{ \pm}\right)} \quad \text { for a.e. } t \geq 0 .
$$

Remark 4.2. It is easy to verify that any $(\eta, u, p)$, which is a solution established in Theorem 3.3 , is a strong solution to the linearized system (1.5)-(1.7).

Theorem 4.1. Assume that $\left(\eta_{1}, v, p_{1}\right)$ and $\left(\eta_{2}, w, p_{2}\right)$ are two strong solutions to (1.5)-(1.7), with $v(0)=w(0)=u_{0}, \eta_{1}(0)=\eta_{2}(0)=\eta_{0}$. Then, $\left(\eta_{1}, v, p_{1}\right)=\left(\eta_{2}, w, p_{2}+c\right)$ for some constant c.

Proof. Let $(\eta, u, p)=\left(\eta_{1}-\eta_{2}, v-w, p_{1}-p_{2}\right)$. Recalling Definition $4.1,(\eta, u, p)$ is still a strong solution to the linearized system (1.5)-(1.7) with zero initial data, i.e. $\eta(0)=0$ and $u(0)=0$.

Multiplying (4.7) by $u$, integrating over $(0, \tau) \times \Omega$ for any $\tau \in\left(0, t_{0}\right)$ and using (4.14), we find that

$$
\int_{0}^{\tau} \int_{\Omega} \varrho \partial_{t} u \cdot u \mathrm{~d} x \mathrm{~d} t+\int_{0}^{\tau} \int_{\Omega} \operatorname{div}\left(p I-\mu\left(\nabla u+\nabla u^{T}\right)\right) \cdot u \mathrm{~d} x \mathrm{~d} t=0 .
$$

Thanks to Lemma 4.1, (4.11)-(4.14) and the regularity of $p$, we obtain

$$
\begin{aligned}
& \int_{0}^{\tau} \int_{\Omega} \operatorname{div}\left(p I-\mu\left(\nabla u+\nabla u^{T}\right)\right) \cdot u \mathrm{~d} x \mathrm{~d} t \\
& =\int_{0}^{\tau} \int_{\mathbb{R}^{2}}\left(\left(\lambda_{-}(p) I-\mu\left(\nabla u_{-}+\nabla u_{-}^{T}\right)\right)-\left(\lambda_{+}(p) I-\mu_{+}\left(\nabla u_{+}+\nabla u_{+}^{T}\right)\right)\right) e_{3} \cdot u \mathrm{~d} x^{\prime} \mathrm{d} t \\
& \quad+\int_{0}^{\tau} \int_{\Omega} \mu \nabla u:\left(\nabla u+\nabla u^{T}\right) \mathrm{d} x \mathrm{~d} t .
\end{aligned}
$$

Notice that in view of (4.6), $p(t) \in\left(H^{1}\left(\Omega_{ \pm}\right)\right)^{3}$ and $u(t) \in\left(H^{2}\left(\Omega_{ \pm}\right)\right)^{3}$ for a.e. $t>0$. Thus, (4.7) implies that

$$
\partial_{t} u \in\left(L^{2}\left(\left(0, t_{0}\right) \times \Omega\right)\right)^{3},
$$

which, together with $u \in L^{\infty}\left(0, t_{0} ;\left(H^{1}(\Omega)\right)^{3}\right) \cap C^{0}\left(\left[0, t_{0}\right],\left(L^{2}(\Omega)\right)^{3}\right)$, yields

$$
\int_{0}^{\tau} \int_{\Omega} \varrho \partial_{t} u \cdot u \mathrm{~d} x \mathrm{~d} t=\frac{1}{2} \int_{\Omega} \varrho u^{2}(\tau) \mathrm{d} x-\frac{1}{2} \int_{\Omega} \varrho u^{2}(0) \mathrm{d} x .
$$

In view of $(4.10),(4.17)-(4.18),(4.19)$ and $u(0)=0$, we find that

$$
\begin{aligned}
& \frac{1}{2} \int_{\Omega} \varrho u^{2}(\tau) \mathrm{d} x+\int_{0}^{\tau} \int_{\Omega} \mu \nabla u:\left(\nabla u+\nabla u^{T}\right) \mathrm{d} x \mathrm{~d} t \\
& =\int_{0}^{\tau} \int_{\mathbb{R}^{2}}\left(g[\varrho] \eta+\kappa \Delta_{x^{\prime}} \eta\right) u_{3} \mathrm{~d} x^{\prime} \mathrm{d} t .
\end{aligned}
$$

Since $\eta \in C^{0}\left(\left[0, t_{0}\right], L_{\text {loc }}^{2}\left(\mathbb{R}^{2}\right)\right)$ and $\eta(0)=0$, the equation (4.9) implies that

$$
\eta\left(t, x^{\prime}\right)=\int_{0}^{t} u_{3}\left(s, x^{\prime}, 0\right) \mathrm{d} s \text { for any } t \geq 0 .
$$

Using (4.15), (4.16), (4.21) and the regularity of $\eta$, we infer that 


$$
\begin{aligned}
\int_{0}^{\tau} \int_{\mathbb{R}^{2}} \Delta_{x^{\prime}} \eta u_{3} \mathrm{~d} x^{\prime} \mathrm{d} t & =-\sum_{i=1}^{2} \int_{0}^{\tau} \int_{\mathbb{R}^{2}} \partial_{i} \eta \partial_{i} u_{3} \mathrm{~d} x^{\prime} \mathrm{d} t \\
& =-\sum_{i=1}^{2} \int_{0}^{\tau} \int_{\mathbb{R}^{2}} \int_{0}^{t} \partial_{i} u_{3}\left(s, x^{\prime}, 0\right) \mathrm{d} s \partial_{i} u_{3}\left(t, x^{\prime}, 0\right) \mathrm{d} x^{\prime} \mathrm{d} t
\end{aligned}
$$

where the formula of integration by parts can be shown in the same manner as in Lemma 4.1. Consequently, inserting (4.21) and (4.22) into (4.20), we arrive at

$$
\begin{aligned}
& \frac{1}{2} \int_{\Omega} \varrho u^{2}(\tau) \mathrm{d} x \mathrm{~d} t+\int_{0}^{\tau} \int_{\Omega} \mu \nabla u:\left(\nabla u+\nabla u^{T}\right) \mathrm{d} x \mathrm{~d} t \\
& =g[\varrho] \int_{0}^{\tau} \int_{\mathbb{R}^{2}} \int_{0}^{t} u_{3}\left(s, x^{\prime}, 0\right) \mathrm{d} s u_{3}\left(t, x^{\prime}, 0\right) \mathrm{d} x^{\prime} \mathrm{d} t \\
& \quad-\kappa \sum_{i=1}^{2} \int_{0}^{\tau} \int_{\mathbb{R}^{2}} \int_{0}^{t} \partial_{i} u_{3}\left(s, x^{\prime}, 0\right) \mathrm{d} s \partial_{i} u_{3}\left(t, x^{\prime}, 0\right) \mathrm{d} x^{\prime} \mathrm{d} t
\end{aligned}
$$

With the help of the regularity of $\partial_{i} u_{3}$, the property of absolutely continuous functions and the Fubini theorem, we conclude that

$$
\begin{aligned}
\int_{0}^{\tau} \int_{\mathbb{R}^{2}} \int_{0}^{t} \partial_{i} u_{3}\left(s, x^{\prime}, 0\right) \mathrm{d} s \partial_{i} u_{3}\left(t, x^{\prime}, 0\right) \mathrm{d} x^{\prime} \mathrm{d} t & =\int_{\mathbb{R}^{2}} \int_{0}^{\tau} \int_{0}^{t} \partial_{i} u_{3}\left(s, x^{\prime}, 0\right) \mathrm{d} s \partial_{i} u_{3}\left(t, x^{\prime}, 0\right) \mathrm{d} t \mathrm{~d} x^{\prime} \\
& =\int_{\mathbb{R}^{2}} \int_{0}^{\tau} \frac{d}{d t}\left[\int_{0}^{t} \partial_{i} u_{3}\left(s, x^{\prime}, 0\right) \mathrm{d} s\right]^{2} \mathrm{~d} t \mathrm{~d} x^{\prime} \\
& =\int_{\mathbb{R}^{2}}\left[\int_{0}^{\tau} \partial_{i} u_{3}\left(t, x^{\prime}, 0\right) \mathrm{d} t\right]^{2} \mathrm{~d} x^{\prime} \geq 0 .
\end{aligned}
$$

On the other hand, applying the Cauchy-Schwarz inequality, we get

$$
2 \sum_{i=1}^{3} \int_{\Omega} \mu\left(\partial_{i} u_{i}\right)^{2} \mathrm{~d} x \mathrm{~d} t \leq \int_{\Omega} \mu \nabla u:\left(\nabla u+\nabla u^{T}\right) \mathrm{d} x \mathrm{~d} t
$$

Hence, by (4.23)-(4.25), we have

$$
\int_{\Omega} \varrho u^{2}(\tau) \mathrm{d} x+4 \sum_{i=1}^{3} \int_{\Omega} \mu\left(\partial_{i} u_{i}\right)^{2} \mathrm{~d} x \mathrm{~d} t \leq 2 g[\varrho] \int_{0}^{\tau} \int_{\mathbb{R}^{2}} \int_{0}^{t} u_{3}\left(s, x^{\prime}, 0\right) \mathrm{d} s u_{3}\left(t, x^{\prime}, 0\right) \mathrm{d} x^{\prime} \mathrm{d} t .
$$

Similarly to (4.24), the right-hand side of (4.26) can be bounded as follows.

$$
\begin{aligned}
& 2 \int_{0}^{\tau} \int_{\mathbb{R}^{2}} \int_{0}^{t} u_{3}\left(s, x^{\prime}, 0\right) u_{3}\left(t, x^{\prime}, 0\right) \mathrm{d} x^{\prime} \mathrm{d} s \mathrm{~d} t=\int_{\mathbb{R}^{2}}\left(\int_{0}^{\tau} u_{3}\left(t, x^{\prime}, 0\right) \mathrm{d} t\right)^{2} \mathrm{~d} x^{\prime} \\
& \leq \tau \int_{0}^{\tau} \int_{\mathbb{R}^{2}}\left|u_{3}\left(t, x^{\prime}, 0\right)\right|^{2} \mathrm{~d} x^{\prime} \mathrm{d} t \\
& =2 \tau \int_{0}^{\tau} \int_{\mathbb{R}^{2}} \int_{1}^{0} u_{3}\left(t, x^{\prime}, x_{3}\right) \partial_{3} u_{3}\left(t, x^{\prime}, x_{3}\right) \mathrm{d} x_{3} \mathrm{~d} x^{\prime} \mathrm{d} t \\
& \leq \tau \int_{0}^{\tau} \int_{\mathbb{R}^{2}}\left(\frac{\mu}{2 \tau g[\varrho]} \int_{0}^{1}\left|\partial_{3} u_{3}(t)\right|^{2} \mathrm{~d} x_{3}+\frac{2 \tau g[\varrho]}{\mu} \int_{0}^{1}\left|u_{3}(t)\right|^{2} \mathrm{~d} x_{3}\right) \mathrm{d} x^{\prime} \mathrm{d} t \\
& \leq \frac{1}{2 g[\varrho]} \int_{0}^{\tau}\left\|\sqrt{\mu} \partial_{3} u_{3}(t)\right\|_{L^{2}(\Omega)}^{2} \mathrm{~d} t+\frac{2 \tau^{2} g[\varrho]}{\mu-} \int_{0}^{\tau}\|u(t)\|_{L^{2}(\Omega)}^{2} \mathrm{~d} t .
\end{aligned}
$$


Substituting (4.27) into (4.26), we deduce

$$
\|\sqrt{\varrho} u(\tau)\|_{L^{2}(\Omega)}^{2}+3 \mu_{-} \int_{0}^{\tau}\|\nabla u(t)\|_{L^{2}(\Omega)}^{2} \mathrm{~d} t \leq 4 \tau^{2} g^{2}[\varrho]^{2} \mu_{-}^{-1} \int_{0}^{\tau}\|u(t)\|_{L^{2}(\Omega)}^{2} \mathrm{~d} t,
$$

which results in

$$
\|u(\tau)\|_{L^{2}(\Omega)}^{2} \leq \frac{4 \tau^{2} g^{2}[\varrho]^{2}}{\mu_{-} \varrho_{-}} \int_{0}^{\tau}\|u(t)\|_{L^{2}(\Omega)}^{2} \mathrm{~d} t .
$$

Moreover, if we apply the Beesack inequality to (4.28), we obtain

$$
\|u(\tau)\|_{L^{2}(\Omega)}^{2}=0 \quad \text { for any } \tau \in\left[0, t_{0}\right]
$$

which implies $u=0$, i.e. $v=w$. This, combined with (4.7) and (4.9), proves Theorem 4.1.

Remark 4.3. In addition, employing arguments similar to those used for (4.1), the regularity of $u$ stated in Remark 4.1 and the fact that $\operatorname{div} u=0$, we can show

$$
\int_{0}^{\tau} \int_{\Omega} \mu \nabla u: \nabla u^{T} \mathrm{~d} x \mathrm{~d} t \equiv 0
$$

\subsection{Proof of Theorem 2.1}

We define

$$
\beta_{1}=\left\{\begin{array}{ll}
|\xi|_{c} / 3, & \text { if } \kappa>0, \\
1, & \text { if } \kappa=0,
\end{array} \text { and } \beta_{2}= \begin{cases}2|\xi|_{c} / 3, & \text { if } \kappa>0 \\
2, & \text { if } \kappa=0\end{cases}\right.
$$

Fix $j \geq k \geq 0, \alpha>0$ and let $\tilde{c}_{j}$ be the constants from Theorem 3.3. For each $n \in \mathbb{N}$, let $t_{n}$ be sufficiently large, so that

$$
\frac{\exp \left(2 t_{n} \lambda_{0}\right)}{\left(1+\beta_{2}^{2}\right)^{j-k+2}}=\alpha^{2} n^{2} \tilde{c}_{j}^{2}
$$

i.e.

where

$$
t_{n}=\frac{\ln \left(\tilde{c}_{j}\left(1+\beta_{2}^{2}\right)^{(j-k+2) / 2}\right)}{\lambda_{0}}+\frac{\ln (\alpha n)}{\lambda_{0}}:=C_{j k}+C_{1} \ln (\alpha n)
$$

$$
\lambda_{0}=\inf _{\xi \in B\left(0, \beta_{2}\right) \backslash B\left(0, \beta_{1}\right)} \lambda(|\xi|)>0 .
$$

Choose $f_{n} \in C_{0}^{\infty}\left(\mathbb{R}^{2}\right)$, such that $\operatorname{supp}\left(f_{n}\right) \subset B\left(0, \beta_{2}\right) \backslash B\left(0, \beta_{1}\right), f_{n}$ is real-valued and radial, and

$$
\int_{\mathbb{R}^{2}}\left(1+|\xi|^{2}\right)^{j+2}\left|f_{n}(|\xi|)\right|^{2} \mathrm{~d} \xi=\frac{1}{\tilde{c}_{j}^{2} n^{2}} .
$$

Now, we can apply Theorem 3.3 with $f=f_{n}, R_{1}=\beta_{1}$ and $R_{2}=\beta_{2}$ to find that $\left(\eta_{n}(t), u_{n}(t)\right.$, $\left.p_{n}(t)\right) \in H^{j}(\Omega)$ solves the problem (1.5)-(1.7). It follows thus from (3.49) and (4.30) that (2.3) holds for all $n$.

Recalling $\operatorname{supp}\left(f_{n}\right) \subset B\left(\beta_{1}, \beta_{2}\right)$ and $\lambda(|\xi|) \geq \lambda_{0}$, we have, after a straightforward calculation and using (4.29) and (3.34), that

$$
\begin{aligned}
\left\|u_{n}(t)\right\|_{H^{k}}^{2} & \geq \int_{\mathbb{R}^{2}}\left(1+|\xi|^{2}\right)^{k} e^{2 t \lambda(|\xi|)}\left|f_{n}(|\xi|)\right|^{2}\left\|v\left(\xi, x_{3}\right)\right\|_{L^{2}(-1,1)}^{2} \mathrm{~d} \xi \\
& \geq \frac{\exp \left(2 t \lambda_{0}\right)}{\left(1+\beta_{2}^{2}\right)^{j-k+2}} \int_{\mathbb{R}^{2}}\left(1+|\xi|^{2}\right)^{j+2}\left|f_{n}(|\xi|)\right|^{2}\left\|v\left(\xi, x_{3}\right)\right\|_{L^{2}(-1,1)}^{2} \mathrm{~d} \xi \\
& =\alpha^{2} n^{2} \tilde{c}_{j}^{2} \int_{\mathbb{R}^{2}}\left(1+|\xi|^{2}\right)^{j+2}\left|f_{n}(|\xi|)\right|^{2} \mathrm{~d} \xi \\
& =\alpha^{2} \quad \text { for any } t \geq t_{n}
\end{aligned}
$$

which implies (2.4) and (2.5). This completes the proof of Theorem 2.1 


\section{Proof of Theorem 2.2}

In this section, we will argue by contradiction to show Theorem 2.2. Therefore, we suppose that the perturbed problem has the global stability of order $k$ for some $k \geq 3$.

\subsection{Regularity under the assumption of the global stability}

Let $\delta, C_{2}>0$ and $F:[0, \delta] \rightarrow \mathbb{R}^{+}$be the constants and function provided by the global stability of order $k$, respectively. Letting $n \in \mathbb{N}$, and applying Theorem 2.1 with this $n, t_{n}=T_{0} / 2$, $k \geq 3$, and $\alpha=2$, we find that $(\tilde{\eta}, \tilde{u}, \tilde{\sigma})$ solves $(1.5)$, such that

$$
\|\tilde{\eta}(0)\|_{H^{k}\left(\mathbb{R}^{2}\right)}+\|\tilde{u}(0)\|_{H^{k}\left(\Omega_{ \pm}\right)}+\|\tilde{\sigma}(0)\|_{H^{k}\left(\Omega_{ \pm}\right)}<n^{-1},
$$

but

$$
\|\tilde{u}(t)\|_{H^{3}\left(\Omega_{ \pm}\right)} \geq 2 \quad \text { for } t \geq T_{0} / 2 .
$$

By the Sobolev embedding theorem, $\tilde{\eta}(0) \equiv \tilde{\eta}\left(0, x^{\prime}\right) \in C_{\text {loc }}^{0,1}\left(\mathbb{R}^{2}\right)$. Employing the Stein extension theorem, there exist two linear operators $E_{+}$and $E_{-}$which map $H^{k}\left(\Omega_{+}\right)$and $H^{k}\left(\Omega_{-}\right)$to $H^{k}\left(\mathbb{R}^{3}\right)$, respectively, such that

$$
E_{+}\left(\tilde{u}_{+}(0)\right)=\tilde{u}_{+}(0) \text { a.e. in } \Omega^{+}, \quad E_{-}\left(\tilde{u}_{-}(0)\right)=\tilde{u}_{-}(0) \text { a.e. in } \Omega^{-},
$$

and

$$
\left\|E_{+}\left(\tilde{u}_{+}(0)\right)\right\|_{H^{k}\left(\mathbb{R}^{3}\right)} \leq c(k)\left\|\tilde{u}_{+}(0)\right\|_{H^{k}\left(\Omega_{+}\right)}, \quad\left\|E_{-}\left(\tilde{u}_{-}(0)\right)\right\|_{H^{k}\left(\mathbb{R}^{3}\right)} \leq c(k)\left\|\tilde{u}_{-}(0)\right\|_{H^{k}\left(\Omega_{-}\right)}
$$

for some constant $c(k)$ depending on $k$. Keeping in mind that $\|\tilde{\eta}(0)\|_{H^{k}\left(\mathbb{R}^{2}\right)}<n^{-1}$, we can apply the embedding theorem to see that there exists a sufficiently small constant $\varepsilon_{0}>0$, such that $\|\varepsilon \tilde{\eta}(0)\|_{L^{\infty}\left(\mathbb{R}^{2}\right)}<1$ for any $\varepsilon \in\left(0, \varepsilon_{0}\right)$. Thus, we define

$$
\begin{aligned}
& \bar{\eta}_{0}^{\varepsilon}:=\varepsilon \tilde{\eta}(0), \\
& \Sigma^{\varepsilon}(0):=\left\{\left(x^{\prime}, x_{3}\right) \in \mathbb{R}^{3} \mid x_{3}=\bar{\eta}_{0}^{\varepsilon}\left(x^{\prime}\right)\right\}, \\
& \Omega_{+}^{\varepsilon}(0):=\left\{\left(x^{\prime}, x_{3}\right) \in \mathbb{R}^{3} \mid \bar{\eta}_{0}^{\varepsilon}\left(x^{\prime}\right)<x_{3}<1\right\}, \\
& \Omega_{-}^{\varepsilon}(0):=\left\{\left(x^{\prime}, x_{3}\right) \in \mathbb{R}^{3} \mid-1<x_{3}<\bar{\eta}_{0}^{\varepsilon}\left(x^{\prime}\right)\right\}, \\
& v^{\varepsilon}(0):=\left\{\begin{array}{l}
E_{+}\left(\tilde{u}_{+}(0)\right), \text { if } x \in \Omega_{+}^{\varepsilon}(0), \\
E_{-}\left(\tilde{u}_{-}(0)\right), \text { if } x \in \Omega_{-}^{\varepsilon}(0) .
\end{array}\right. \\
& \bar{u}_{0}^{\varepsilon}:=\varepsilon v^{\varepsilon}(0) .
\end{aligned}
$$

Now, we fix $n \in \mathbb{N}$ so that $n>\max \left\{1, C_{2}\right\} \max \{1, c(k)\}$. By construction, $\left.\left[\bar{u}_{0}^{\varepsilon}\right]\right|_{\Sigma^{\varepsilon}(0)}=0$. Moreover, for $\varepsilon<\tilde{\varepsilon}_{0}:=\min \left\{\varepsilon_{0}, \delta n(\max \{1, c(k)\})^{-1}\right\}$, we have

$$
\begin{aligned}
& \left\|\bar{\eta}_{0}^{\varepsilon}\right\|_{H^{k}\left(\mathbb{R}^{2}\right)}+\left\|\bar{u}_{0}^{\varepsilon}\right\|_{H^{k}\left(\Omega_{ \pm}^{\varepsilon}(0)\right)} \\
& =\varepsilon\left(\|\tilde{\eta}(0)\|_{H^{k}\left(\mathbb{R}^{2}\right)}+\sqrt{\left\|E_{+}\left(\tilde{u}_{+}(0)\right)\right\|_{H^{k}\left(\Omega_{+}^{\varepsilon}(0)\right)}^{2}+\left\|E_{-}\left(\tilde{u}_{-}(0)\right)\right\|_{H^{k}\left(\Omega_{-}^{\varepsilon}(0)\right)}^{2}}\right) \\
& <\varepsilon\left(\|\tilde{\eta}(0)\|_{H^{k}\left(\mathbb{R}^{2}\right)}+c(k) \sqrt{\left\|\tilde{u}_{+}(0)\right\|_{H^{k}\left(\Omega_{+}\right)}^{2}+\left\|\tilde{u}_{-}(0)\right\|_{H^{k}\left(\Omega_{-}\right)}^{2}}\right) \\
& \leq \varepsilon \max \{1, c(k)\}\left(\|\tilde{\eta}(0)\|_{H^{k}\left(\mathbb{R}^{2}\right)}+\|\tilde{u}(0)\|_{H^{k}\left(\Omega_{ \pm}\right)}\right)<\delta .
\end{aligned}
$$

Thus, according to the global stability of order $k$, there exist $\eta^{\varepsilon}, u^{\varepsilon}, \sigma^{\varepsilon}$ in the function class described in Definition 2.1, which solve the perturbed problem:

$$
\left\{\begin{array}{l}
\partial_{t} \eta^{\varepsilon}=u_{3}^{\varepsilon}-u_{1}^{\varepsilon} \partial_{1} \eta^{\varepsilon}-u_{2}^{\varepsilon} \partial_{2} \eta^{\varepsilon} \\
\varrho \partial_{t} u^{\varepsilon}+\varrho\left(\nabla u^{\varepsilon}\right) u^{\varepsilon}+\nabla \sigma^{\varepsilon}=\mu \Delta u^{\varepsilon} \\
\operatorname{div} u^{\varepsilon}=0
\end{array}\right.
$$


with the jump condition

$$
\begin{aligned}
& {\left.\left[u^{\varepsilon}\right]\right|_{\Sigma^{\varepsilon}(t)}=0,} \\
& {\left.\left[\left(\sigma^{\varepsilon} I-\mu\left(\nabla u^{\varepsilon}+\nabla\left(u^{\varepsilon}\right)^{T}\right)\right) \nu^{\varepsilon}\right]\right|_{\Sigma^{\varepsilon}(t)}=\left(g[\varrho] \eta^{\varepsilon}+\kappa H^{\varepsilon}\right) \nu^{\varepsilon},} \\
& \Sigma^{\varepsilon}(t):=\left\{x \in \mathbb{R}^{3} \mid x_{3}=\eta^{\varepsilon}\left(t, x^{\prime}\right)\right\},
\end{aligned}
$$

and the initial data satisfying $\left\|\bar{\eta}_{0}^{\varepsilon}\right\|_{H^{k}\left(\mathbb{R}^{2}\right)}+\left\|\bar{u}_{0}^{\varepsilon}\right\|_{H^{k}\left(\Omega_{ \pm}^{\varepsilon}(0)\right)}<\delta$. Furthermore, the solution satisfies

$$
\begin{aligned}
& \sup _{0 \leq t<\infty}\left(\left\|u^{\varepsilon}\right\|_{H^{3}\left(\Omega_{ \pm}^{\varepsilon}(t)\right)}+\left\|\eta^{\varepsilon}\right\|_{H^{2}\left(\mathbb{R}^{2}\right)}+\left\|\sigma^{\varepsilon}\right\|_{H^{1}\left(\Omega_{ \pm}^{\varepsilon}(t)\right)}\right) \leq F\left(\left\|\bar{\eta}_{0}^{\varepsilon}\right\|_{H^{k}\left(\mathbb{R}^{2}\right)}+\left\|\bar{u}_{0}^{\varepsilon}\right\|_{H^{k}\left(\Omega_{ \pm}^{\varepsilon}(0)\right)}\right) \\
& \quad \leq \varepsilon C_{2} \max \{1, c(k)\}\left(\|\tilde{\eta}(0)\|_{H^{k}\left(\mathbb{R}^{2}\right)}+\|\tilde{u}(0)\|_{H^{k}\left(\Omega_{ \pm}\right)}\right)<\varepsilon .
\end{aligned}
$$

Now, define the rescaled functions $\bar{\eta}^{\varepsilon}=\eta^{\varepsilon} / \varepsilon, \bar{u}^{\varepsilon}=u^{\varepsilon} / \varepsilon, \bar{\sigma}^{\varepsilon}=\sigma^{\varepsilon} / \varepsilon$. If we rescale (5.6), then we see that

$$
\sup _{0 \leq t<\infty}\left(\left\|\bar{u}^{\varepsilon}(t)\right\|_{H^{3}\left(\Omega_{ \pm}^{\varepsilon}(t)\right)}+\left\|\bar{\eta}^{\varepsilon}(t)\right\|_{H^{2}\left(\mathbb{R}^{2}\right)}+\left\|\bar{\sigma}^{\varepsilon}(t)\right\|_{H^{1}\left(\Omega_{ \pm}^{\varepsilon}(t)\right)}\right) \leq 1,
$$

where

$$
\begin{aligned}
& \Omega_{+}^{\varepsilon}(t)=\left\{\left(x^{\prime}, x_{3}\right) \mid x^{\prime} \in \mathbb{R}^{2}, \eta^{\varepsilon}\left(t, x^{\prime}\right)<x_{3}<1\right\}, \\
& \Omega_{-}^{\varepsilon}(t)=\left\{\left(x^{\prime}, x_{3}\right) \mid x^{\prime} \in \mathbb{R}^{2},-1<x_{3}<\eta^{\varepsilon}\left(t, x^{\prime}\right)\right\}, \quad t>0 .
\end{aligned}
$$

Moreover, recalling the definition of $H^{3}\left(\Omega_{ \pm}^{\varepsilon}(t)\right)$ in (2.2), using (5.4) and the regularity of $\bar{u}^{\varepsilon}(t)$ in (5.7), we can easily verify that

$$
\sup _{0 \leq t<\infty}\left\|\bar{u}^{\varepsilon}(t)\right\|_{H_{0}^{1}(\Omega)} \leq 1 .
$$

The equations $(5.3)_{2}$, together with (5.7), yield

$$
\begin{aligned}
\sup _{0 \leq t<\infty}\left\|\partial_{t} \bar{u}^{\varepsilon}\right\|_{L^{2}(\Omega)} & \leq \sqrt{5}\left(\varepsilon\left\|\bar{u}^{\varepsilon}\right\|_{L^{2}\left(\Omega_{ \pm}^{\varepsilon}(t)\right)}\left\|\nabla \bar{u}^{\varepsilon}\right\|_{L^{2}\left(\Omega_{ \pm}^{\varepsilon}(t)\right)}+\varrho_{-}^{-1}\left\|\nabla \bar{\sigma}^{\varepsilon}\right\|_{L^{2}\left(\Omega_{ \pm}^{\varepsilon}(t)\right)}+\mu_{+} \varrho_{-}^{-1}\left\|\Delta \bar{u}^{\varepsilon}\right\|_{L^{2}\left(\Omega_{ \pm}^{\varepsilon}(t)\right)}\right) \\
& \leq \sqrt{5}\left[\delta n+\left(1+\mu_{+}\right) / \varrho_{-}\right] .
\end{aligned}
$$

Now, letting $j \in \mathbb{Z}^{+}$, and employing (5.3), (5.7) and (5.8), we infer that for each square domain,

$$
\mathcal{R}_{j}:=\left\{x^{\prime} \in \mathbb{R}^{2}|| x_{1}|,| x_{2} \mid<j\right\},
$$

there exists a constant $c_{1}(j)$ depending on $j$, such that

$$
\sup _{0 \leq t<\infty}\left\|\partial_{t} \bar{\eta}^{\varepsilon}\right\|_{L^{2}\left(\mathcal{R}_{j}\right)} \leq c_{1}(j)
$$

\subsection{Taking limits in the rescaled function sequences}

First, making use of (5.7)-(5.10), an abstract version of the Arzela-Ascoli theorem (see [13, Theorem 1.70]), and the Aubin-Lions Theorem (see [13, Theorem 1.71]), we can extract a subsequence $\left(\bar{\eta}_{m}, \bar{u}_{m}\right):=\left(\bar{\eta}^{\varepsilon_{m}}, \bar{u}^{\varepsilon_{m}}\right)$, with $\left\{\varepsilon_{m}\right\} \subset\left\{\varepsilon \mid 0<\varepsilon<\tilde{\varepsilon}_{0}\right\}$, such that, for any $p \geq 1$ and $j \in \mathbb{Z}^{+}$,

$$
\begin{aligned}
\bar{u}_{m} \rightarrow & \bar{u} \text { weakly star in } L^{\infty}\left(0, T_{0} ; H_{0}^{1}(\Omega)\right), \\
& \text { and strongly in } C^{0}\left(\left[0, T_{0}\right], L^{2}(\Omega)\right), \\
\partial_{t} \bar{u}_{m} \rightarrow & \partial_{t} \bar{u} \text { weakly star in } L^{\infty}\left(0, T_{0} ; L^{2}(\Omega)\right), \\
\bar{\eta}_{m} \rightarrow & \bar{\eta} \text { weakly star in } L^{\infty}\left(0, T_{0} ; H^{2}\left(\mathbb{R}^{2}\right)\right), \\
& \quad \text { and strongly in } C^{0}\left(\left[0, T_{0}\right], L^{2}\left(\mathcal{R}_{j}\right)\right) \cap L^{p}\left(0, T_{0} ; H^{1}\left(\mathcal{R}_{j}\right)\right), \\
\partial_{t} \bar{\eta}_{m} \rightarrow & \partial_{t} \bar{\eta} \text { weakly star in } L^{\infty}\left(0, T_{0} ; L^{2}\left(\mathcal{R}_{j}\right)\right), \\
\bar{\sigma}_{m} \rightarrow & \bar{\sigma} \text { weakly star in } L^{\infty}\left(0, T_{0} ; L^{2}(\Omega)\right) .
\end{aligned}
$$


Hereafter, for simplicity we denote $f^{\varepsilon_{m}}$ by $f_{m}$, where $f$ represents $\bar{u}, \bar{\eta}, \bar{\sigma}, \Sigma$, or $\eta$, and so on.

Denoting

$$
\Omega_{ \pm}^{j}=\left\{\left(x^{\prime}, x_{3}\right) \in \Omega_{ \pm}|1 / j<| x_{3} \mid<1\right\},
$$

and using the regularity of $\eta^{\varepsilon}$ in (5.6) and the Sobolev embedding theorem, we find that for any positive integer $j$, there exists an $\varepsilon_{m_{j}}>0$ depending on $j$, such that, for any $\varepsilon_{m} \in\left(0, \varepsilon_{m_{j}}\right)$ and $t \geq 0$, we have

$$
\Omega_{ \pm}^{j} \subset\left\{\left(x^{\prime}, x_{3}\right) \in \Omega_{ \pm}|| \eta_{m}\left(t, x^{\prime}\right)|<| x_{3} \mid<1\right\} \subset \Omega .
$$

Therefore, for any $j$, making use of (5.7), (5.9), (5.17), and the Lions-Aubin Lemma, we can show by induction that there exists $\left\{m_{i}^{j}\right\} \subset\left\{m_{i}^{j-1}\right\} \subset\{m\}$ such that,

$$
\begin{aligned}
& \varepsilon_{m_{i}^{j}}<\varepsilon_{m_{j}}, \text { for any } i>0, \\
& \bar{u}_{m_{i}^{j}} \rightarrow \bar{u} \text { weakly star in } L^{\infty}\left(0, T_{0} ; H^{3}\left(\Omega_{ \pm}^{j}\right)\right) \\
& \quad \text { and strongly in } L^{p}\left(0, T_{0} ; H^{2}\left(\Omega_{ \pm}^{j}\right)\right) \text { for any } p \geq 1, \\
& \bar{\sigma}_{m_{i}^{j}} \rightarrow \bar{\sigma} \text { weakly star in } L^{\infty}\left(0, T_{0} ; H^{1}\left(\Omega_{ \pm}^{j}\right)\right), \quad \text { as } i \rightarrow \infty,
\end{aligned}
$$

where we have defined $\left\{m_{i}^{0}\right\}=\{m\}$.

From the lower semicontinuity, one gets

$$
\sup _{0 \leq t<T_{0}}\left(\|\bar{u}(t)\|_{H^{3}\left(\Omega_{ \pm}^{j}\right)}+\|\bar{\eta}(t)\|_{H^{2}\left(\mathbb{R}^{2}\right)}+\|\bar{\sigma}(t)\|_{H^{1}\left(\Omega_{ \pm}^{j}\right)}\right) \leq 1 .
$$

Choosing $m_{i}^{\prime}=m_{i}^{i}$, one has, for any $j \in \mathbb{N}$ and $i>j$, that

$$
\begin{array}{r}
\bar{u}_{m_{i}^{\prime}} \rightarrow \bar{u} \text { weakly star in } L^{\infty}\left(0, T_{0} ; H^{3}\left(\Omega_{ \pm}^{j}\right)\right), \\
\quad \text { and strongly in } L^{p}\left(0, T_{0} ; H^{2}\left(\Omega_{ \pm}^{j}\right)\right), \quad \text { for any } p \geq 1, \\
\bar{\sigma}_{m_{i}^{\prime}} \rightarrow \bar{\sigma} \text { weakly star in } L^{\infty}\left(0, T_{0} ; H^{1}\left(\Omega_{ \pm}^{j}\right)\right), \quad \text { as } i \rightarrow \infty .
\end{array}
$$

Moreover, by (5.13) and (5.18)-(5.19), we find that

$$
\left\{\begin{array}{l}
\varrho \partial_{t} \bar{u}+\nabla \bar{\sigma}=\mu \Delta \bar{u} \\
\operatorname{div} \bar{u}=0
\end{array}\right.
$$

holds a.e. in $\left(0, T_{0}\right) \times\left(\Omega \backslash\left\{x_{3}=0\right\}\right)$, and

$$
\sup _{0 \leq t<T_{0}}\left(\|\bar{u}(t)\|_{H^{3}\left(\Omega_{ \pm}\right)}+\|\bar{\eta}(t)\|_{H^{2}\left(\mathbb{R}^{2}\right)}+\|\bar{\sigma}(t)\|_{H^{1}\left(\Omega_{ \pm}\right)}\right) \leq 1 .
$$

In addition, by construction, we have

$$
\begin{aligned}
& \bar{\eta}^{\varepsilon}(0)=\tilde{\eta}(0):=\tilde{\eta}\left(0, x^{\prime}\right) \text { in } \mathbb{R}^{2}, \\
& \bar{u}_{m_{i}^{\prime}}(0)=\tilde{u}(0) \quad \text { in } \Omega_{ \pm}^{j} \quad \text { for any } i>j .
\end{aligned}
$$

The estimates (5.20) and (5.22), combined with (5.12) and (5.15), imply

$$
\bar{\eta}(0)=\tilde{\eta}(0) \quad \text { and } \quad \bar{u}(0)=\tilde{u}(0) .
$$

5.3. Convergence of the interface equation

Replacing $\varepsilon$ by $\varepsilon_{m}$, we rewrite $(5.3)_{1}$ as

$$
\bar{\eta}_{m}=\left(\bar{v}_{m, 3}-\varepsilon_{m} \bar{v}_{m, 1} \partial_{1} \bar{\eta}_{m}-\varepsilon_{m} \bar{v}_{m, 2} \partial_{2} \bar{\eta}_{m}\right),
$$

where 


$$
\bar{v}_{m}:=\bar{v}_{m}\left(t, x^{\prime}\right)=\bar{u}_{m}\left(t, x^{\prime}, \eta_{m}\left(t, x^{\prime}\right)\right), \quad t \in\left(0, T_{0}\right),
$$

and $\bar{v}_{m, 1}, \bar{v}_{m, 2}$ denote the first and second components of the vector function $\bar{v}_{m}$, respectively.

Multiplying (5.24) with $\varphi \in \mathcal{D}\left(\mathbb{R}^{2}\right)$, and integrating over $\mathbb{R}^{2}$, then we arrive at

$$
\int_{\mathbb{R}^{2}} \partial_{t} \bar{\eta}_{m} \varphi \mathrm{d} x^{\prime}=\int_{\mathbb{R}^{2}}\left(\bar{v}_{m, 3}-\varepsilon_{m} \bar{v}_{m, 1} \partial_{1} \bar{\eta}_{m}-\varepsilon_{m} \bar{v}_{m, 2} \partial_{2} \bar{\eta}_{m}\right) \varphi \mathrm{d} x^{\prime}
$$

Recalling $\bar{\eta}_{m} \in C^{0}\left(\left[0, T_{0}\right], L^{2}\left(\mathcal{R}_{j}\right)\right)$, we use (5.22) and (5.25) to deduce that

$$
\int_{\mathbb{R}^{2}} \bar{\eta}_{m}(t) \varphi \mathrm{d} x^{\prime}=\int_{0}^{t}\left[\int_{\mathbb{R}^{2}}\left(\bar{v}_{m, 3}-\varepsilon_{m} \bar{v}_{m, 1} \partial_{1} \bar{\eta}_{m}-\varepsilon_{m} \bar{v}_{m, 2} \partial_{2} \bar{\eta}_{m}\right) \varphi \mathrm{d} x^{\prime}+\int_{\mathbb{R}^{2}} \tilde{\eta}\left(0, x^{\prime}\right) \varphi \mathrm{d} x^{\prime}\right] \mathrm{d} s .
$$

Next, we analyze the convergence of each integral in (5.26).

First, there exists a square domain $\mathcal{R}_{j_{1}}$ satisfying

$$
\operatorname{supp} \varphi \subset \mathcal{R}_{j_{1}} \subset \mathbb{R}^{2} \text {. }
$$

Due to (5.15), we get

$$
\lim _{m \rightarrow \infty} \int_{\mathbb{R}^{2}} \bar{\eta}_{m} \varphi \mathrm{d} x^{\prime}=\lim _{m \rightarrow \infty} \int_{\mathcal{R}_{j_{1}}} \bar{\eta}_{m} \varphi \mathrm{d} x^{\prime}=\int_{\mathcal{R}_{j_{1}}} \bar{\eta} \varphi \mathrm{d} x^{\prime}=\int_{\mathbb{R}^{2}} \bar{\eta} \varphi \mathrm{d} x^{\prime} .
$$

Then, from (5.7) we get

$$
\sup _{0 \leq t<\infty}\left\|\bar{\eta}_{m}(t)\right\|_{H^{2}\left(\mathbb{R}^{2}\right)} \leq 1
$$

Noticing that $\bar{u}_{m, i}(t) \in C^{0}(\bar{\Omega}) \cap H_{0}^{1}(\Omega)$ for $t>0$, we utilize the Hölder inequality, (5.28) and (5.8) to obtain

$$
\begin{aligned}
& \left|\int_{0}^{t} \int_{\mathbb{R}^{2}} \bar{v}_{m, i} \partial_{i} \bar{\eta}_{m} \varphi \mathrm{d} x^{\prime} \mathrm{d} s\right| \leq \sqrt{2}\|\varphi\|_{\infty} \int_{0}^{t}\left(\int_{\mathbb{R}^{2}} \int_{\eta_{m}\left(t, x^{\prime}\right)}^{1}\left|\partial_{3} \bar{u}_{m, i}\right|^{2} \mathrm{~d} x_{3} \mathrm{~d} x^{\prime}\right)^{\frac{1}{2}}\left(\int_{\mathbb{R}^{2}}\left|\partial_{i} \bar{\eta}_{m}\right|^{2} \mathrm{~d} x^{\prime}\right)^{\frac{1}{2}} \mathrm{~d} s \\
& \quad \leq \sqrt{2}\|\varphi\|_{\infty}\left\|\partial_{3} \bar{u}_{m, i}\right\|_{L^{1}\left(\left(0, T_{0}\right), L^{2}(\Omega)\right)} \sup _{0 \leq t<\infty}\left\|\partial_{i} \bar{\eta}_{m}(t)\right\|_{L^{2}\left(\mathbb{R}^{2}\right)}<\sqrt{2}\|\varphi\|_{\infty} T_{0},
\end{aligned}
$$

where $i=1,2$, and

$$
\|\varphi\|_{\infty}:=\sup _{x^{\prime} \in \mathbb{R}^{2}}\left|\varphi\left(x^{\prime}\right)\right|>0 .
$$

Hence, from (5.29) it follows that

$$
\lim _{m \rightarrow \infty} \int_{0}^{t} \int_{\mathbb{R}^{2}}\left(\varepsilon_{m} \bar{v}_{m, 1} \partial_{1} \bar{\eta}_{m}-\varepsilon_{m} \bar{v}_{m, 2} \partial_{2} \bar{\eta}_{m}\right) \varphi \mathrm{d} x^{\prime} \mathrm{d} s=0 .
$$

Finally, keeping in mind that $\left\{m_{i}^{\prime}\right\} \subset\{m\}$ and $\partial_{3} \bar{u}_{3} \in L^{\infty}\left(0, T_{0} ; L^{2}(\Omega)\right)$, we use (5.8), (5.18) and the absolute continuity of integrals to deduce that for any $\delta>0$, there exists a $j_{2}>j_{1}>0$ depending on $j_{1}, T_{0}$ and $\|\varphi\|_{\infty}$, such that for any $i>j_{2}$,

$$
\begin{aligned}
& \int_{0}^{T_{0}} \int_{\mathcal{R}_{j_{1}}} \int_{-j_{2}^{-1}}^{j_{2}^{-1}}\left|\partial_{3} \bar{u}_{3}\right| \mathrm{d} x_{3} \mathrm{~d} x^{\prime} \mathrm{d} s<\frac{\delta}{3\|\varphi\|_{\infty}}, \\
& \int_{0}^{T_{0}} \int_{\mathcal{R}_{j_{1}}} \int_{-j_{2}^{-1}}^{j_{2}^{-1}}\left|\partial_{3} \bar{u}_{m_{i}^{\prime}, 3}-\partial_{3} \bar{u}_{3}\right| \mathrm{d} x_{3} \mathrm{~d} x^{\prime}<\frac{\delta}{3\|\varphi\|_{\infty}} \\
& \int_{0}^{T_{0}} \int_{\mathcal{R}_{j_{1}}} \int_{j_{2}^{-1}}^{1}\left|\partial_{3} \bar{u}_{m_{i}^{\prime}, 3}-\partial_{3} \bar{u}_{3}\right| \mathrm{d} x_{3} \mathrm{~d} x^{\prime}<\frac{\delta}{3\|\varphi\|_{\infty}}
\end{aligned}
$$


which, recalling $\left\|\eta_{m_{i}^{\prime}}\left(t, x^{\prime}\right)\right\|_{L^{\infty}\left(\mathbb{R}^{2}\right)}<j_{2}^{-1}$ by the construction of $\left\{m_{i}^{\prime}\right\}$ and (5.17), imply

$$
\begin{aligned}
& \left|\int_{0}^{t} \int_{\mathbb{R}^{2}}\left(\bar{v}_{m_{i}^{\prime}, 3}-\bar{u}_{3}\left(s, x^{\prime}, 0\right)\right) \varphi \mathrm{d} x^{\prime} \mathrm{d} s\right| \\
& =\left|\int_{0}^{t} \int_{\mathbb{R}^{2}}\left[\int_{\eta_{m_{i}^{\prime}}\left(t, x^{\prime}\right)}^{1}\left(\partial_{3} \bar{u}_{m_{i}^{\prime}, 3}-\partial_{3} \bar{u}_{3}\right) \mathrm{d} x_{3}+\int_{\eta_{m_{i}^{\prime}}\left(t, x^{\prime}\right)}^{0} \partial_{3} \bar{u}_{3} \mathrm{~d} x_{3}\right] \varphi \mathrm{d} x^{\prime} \mathrm{d} s\right| \\
& \leq\|\varphi\|_{\infty}\left[\int_{0}^{T_{0}} \int_{\mathcal{R}_{j_{1}}} \int_{\eta_{m_{i}^{\prime}}\left(t, x^{\prime}\right)}^{1}\left|\partial_{3} \bar{u}_{m_{i}^{\prime}, 3}-\partial_{3} \bar{u}_{3}\right| \mathrm{d} x_{3} \mathrm{~d} x^{\prime} \mathrm{d} s+\int_{0}^{T_{0}} \int_{\mathcal{R}_{j_{1}}}\left|\int_{\eta_{m_{i}^{\prime}}\left(t, x^{\prime}\right)}^{0} \partial_{3} \bar{u}_{3} \mathrm{~d} x_{3}\right| \mathrm{d} x^{\prime} \mathrm{d} s\right] \\
& \leq\|\varphi\|_{\infty}\left(\int_{0}^{T_{0}} \int_{\mathcal{R}_{j_{1}}} \int_{j_{2}^{-1}}^{1}\left|\partial_{3} \bar{u}_{m_{i}^{\prime}, 3}-\partial_{3} \bar{u}_{3}\right| \mathrm{d} x_{3} \mathrm{~d} x^{\prime} \mathrm{d} s\right. \\
& \left.\quad+\int_{0}^{T_{0}} \int_{\mathcal{R}_{j_{1}}}^{j_{-j_{2}}^{-1}}\left|\partial_{3} \bar{u}_{m_{i}^{\prime}, 3}-\partial_{3} \bar{u}_{3}\right| \mathrm{d} x_{3} \mathrm{~d} x^{\prime} \mathrm{d} s+\int_{0}^{T_{0}} \int_{\mathcal{R}_{j_{1}}} \int_{-j_{2}^{-1}}^{j_{2}^{-1}}\left|\partial_{3} \bar{u}_{3}\right| \mathrm{d} x_{3} \mathrm{~d} x^{\prime} \mathrm{d} s\right)<\delta,
\end{aligned}
$$

whence,

$$
\lim _{i \rightarrow \infty} \int_{0}^{t} \int_{\mathbb{R}^{2}} \bar{v}_{m_{i}^{\prime}, 3} \varphi \mathrm{d} x^{\prime} \mathrm{d} s=\int_{0}^{t} \int_{\mathbb{R}^{2}} \bar{u}_{3}\left(t, x^{\prime}, 0\right) \varphi \mathrm{d} x^{\prime} \mathrm{d} s .
$$

Consequently, letting $i \rightarrow \infty$, then $m_{i}^{\prime} \rightarrow \infty$ in (5.26) (i.e. $\varepsilon_{m_{i}^{\prime}} \rightarrow 0$ with $\varepsilon_{m_{i}^{\prime}}$ in place of $\varepsilon_{m}$ ), we conclude, with the help of (5.27), (5.30) and (5.31), that

$$
\int_{\mathbb{R}^{2}} \bar{\eta} \varphi \mathrm{d} x^{\prime}=\int_{0}^{t} \int_{\mathbb{R}^{2}} \bar{u}_{3}\left(s, x^{\prime}, 0\right) \varphi \mathrm{d} x^{\prime} \mathrm{d} s+\int_{\mathbb{R}^{2}} \tilde{\eta}\left(0, x^{\prime}\right) \varphi \mathrm{d} x^{\prime},
$$

which implies that for a.e. $t \in\left(0, T_{0}\right)$,

$$
\partial_{t} \bar{\eta}=\bar{u}_{3} \text { a.e. in } \mathbb{R}^{2} .
$$

\subsection{Convergence of the momentum equations}

Multiplying $(5.3)_{2}$ by $\phi=\left(\phi_{1}, \phi_{2}, \phi_{3}\right) \in\left(\mathcal{D}\left(\left(0, T_{0}\right) \times \Omega\right)\right)^{3}$ with $\varepsilon_{m}$ in place of $\varepsilon$, integrating over $\left(0, T_{0}\right) \times \Omega$, and using the jump conditions (5.5), we deduce

$$
\begin{aligned}
& \int_{0}^{T_{0}} \quad \int_{\Omega}\left(\varrho \partial_{t} \bar{u}_{m} \cdot \phi+\varepsilon_{m} \varrho\left(\nabla \bar{u}_{m}\right) \bar{u}_{m} \cdot \phi\right) \mathrm{d} x \mathrm{~d} t+\int_{0}^{T_{0}} \int_{\Omega}\left(\mu\left(\nabla \bar{u}_{m}+\nabla \bar{u}_{m}^{T}\right)-\bar{\sigma}_{m} I\right): \nabla \phi \mathrm{d} x \mathrm{~d} t \\
& \quad=g[\varrho] \int_{0}^{T_{0}} \int_{\Sigma_{m}(t)} \bar{\eta}_{m} \varphi_{m} \cdot \nu_{m} \mathrm{~d} S \mathrm{~d} t+\kappa \int_{0}^{T_{0}} \int_{\Sigma_{m}(t)} \bar{H}_{m} \varphi_{m} \cdot \nu_{m} \mathrm{~d} S \mathrm{~d} t,
\end{aligned}
$$

where

$$
\begin{aligned}
& \Sigma_{m}(t):=\left\{\left(x^{\prime}, x_{3}\right) \in \mathbb{R}^{3} \mid x_{3}=\eta_{m}\left(t, x^{\prime}\right)\right\} \quad \text { for each } t>0, \\
& \varphi_{m}:=\varphi_{m}\left(t, x^{\prime}\right)=\phi\left(t, x^{\prime}, \eta_{m}\left(t, x^{\prime}\right)\right), \\
& \nu_{m}=\frac{\left(-\partial_{1} \eta_{m},-\partial_{2} \eta_{m}, 1\right)^{T}}{\sqrt{\left|\partial_{1} \eta_{m}\right|^{2}+\left|\partial_{2} \eta_{m}\right|^{2}+1}}, \\
& \bar{H}_{m}=\frac{\Delta_{x^{\prime}} \bar{\eta}_{m}+\left(\partial_{1} \eta_{m}\right)^{2} \partial_{2}^{2} \bar{\eta}_{m}+\left(\partial_{2} \eta_{m}\right)^{2} \partial_{1}^{2} \bar{\eta}_{m}-2 \partial_{1} \bar{\eta}_{m} \partial_{2} \eta_{m} \partial_{1} \partial_{2} \eta_{m}}{\left(1+\left(\partial_{1} \eta_{m}\right)^{2}+\left(\partial_{2} \eta_{m}\right)^{2}\right)^{3 / 2}} .
\end{aligned}
$$

By virtue of (5.11)-(5.13) and (5.16),

$$
\begin{aligned}
\lim _{m \rightarrow \infty} & {\left[\int_{0}^{T_{0}} \int_{\Omega}\left(\varrho \partial_{t} \bar{u}_{m} \cdot \phi+\varepsilon_{m} \varrho\left(\nabla \bar{u}_{m}\right) \bar{u}_{m} \cdot \phi\right) \mathrm{d} x \mathrm{~d} t+\int_{0}^{T_{0}} \int_{\Omega}\left(\mu\left(\nabla \bar{u}_{m}+\nabla \bar{u}_{m}^{T}\right)-\bar{\sigma}_{m} I\right): \nabla \phi \mathrm{d} x \mathrm{~d} t\right] } \\
= & \int_{0}^{T_{0}} \int_{\Omega} \varrho \partial_{t} \bar{u} \cdot \phi+\int_{0}^{T_{0}} \int_{\Omega}\left(\mu\left(\nabla \bar{u}+\nabla \bar{u}^{T}\right)-\bar{\sigma} I\right): \nabla \phi \mathrm{d} x \mathrm{~d} t .
\end{aligned}
$$


Next, we analyze the convergence of the terms on the right-hand side of (5.32).

(i) Recalling $\mathrm{d} S=\sqrt{\left|\partial_{1} \eta_{m}\right|^{2}+\left|\partial_{2} \eta_{m}\right|^{2}+1} \mathrm{~d} x^{\prime}$, we use the formula of surface integral and (5.33)-(5.35) to infer that

$$
\int_{0}^{T_{0}} \int_{\Sigma_{m}(t)} \bar{\eta}_{m} \varphi_{m} \cdot \nu_{m} \mathrm{~d} S \mathrm{~d} t=\int_{0}^{T_{0}} \int_{\mathbb{R}^{2}} \bar{\eta}_{m}\left(\varphi_{m, 3}-\varphi_{m, 1} \partial_{1} \eta_{m}-\varphi_{m, 2} \partial_{2} \eta_{m}\right) \mathrm{d} x^{\prime} \mathrm{d} t
$$

From the Hölder inequality and (5.28) it follows that

$$
\begin{aligned}
\int_{0}^{T_{0}} \int_{\mathbb{R}^{2}} \bar{\eta}_{m} \varphi_{m, i} \partial_{i} \eta_{m} \mathrm{~d} x^{\prime} \mathrm{d} t & =\varepsilon_{m} \int_{0}^{T_{0}} \int_{\mathbb{R}^{2}} \bar{\eta}_{m} \varphi_{m, i} \partial_{i} \bar{\eta}_{m} \mathrm{~d} x^{\prime} \mathrm{d} t \\
& \leq \varepsilon_{m} T_{0}\|\phi\|_{\infty} \sup _{0 \leq t \leq T_{0}}\left(\left\|\bar{\eta}_{m}\right\|_{L^{2}\left(\mathbb{R}^{2}\right)}\left\|\partial_{i} \bar{\eta}_{m}\right\|_{L^{2}\left(\mathbb{R}^{2}\right)}\right) \\
& \leq \varepsilon_{m} T_{0}\|\phi\|_{\infty}, \quad i=1,2,
\end{aligned}
$$

whence,

$$
\lim _{m \rightarrow \infty} \int_{0}^{T_{0}} \int_{\mathbb{R}^{2}} \bar{\eta}_{m}\left(\varphi_{m, 1} \partial_{1} \eta_{m}+\varphi_{m, 2} \partial_{2} \eta_{m}\right) \mathrm{d} x^{\prime} \mathrm{d} t=0
$$

Denoting

$$
\|\phi\|_{\infty}:=\sup _{(t, x) \in\left(0, T_{0}\right) \times \Omega}|\phi(t, x)|
$$

and noticing that

$$
\begin{aligned}
& \int_{0}^{T_{0}} \int_{\mathbb{R}^{2}}\left|\bar{\eta}_{m}\left(\varphi_{m, 3}-\phi_{3}\left(t, x^{\prime}, 0\right)\right)\right| \mathrm{d} x^{\prime} \mathrm{d} t \\
& \quad \leq \sup _{t \in\left(0, T_{0}\right)}\left\|\bar{\eta}_{m}\right\|_{L^{2}\left(\mathbb{R}^{2}\right)} \int_{0}^{T_{0}}\left[\int_{\mathbb{R}^{2}}\left|\int_{0}^{\eta_{m}\left(t, x^{\prime}\right)} \partial_{3} \phi_{3} \mathrm{~d} x_{3}\right|^{2} \mathrm{~d} x^{\prime}\right]^{1 / 2} \mathrm{~d} t \\
& \quad \leq T_{0} \sup _{t \in\left(0, T_{0}\right)}\left\|\bar{\eta}_{m}\right\|_{L^{2}\left(\mathbb{R}^{2}\right)}\left\|\partial_{3} \phi_{3}\right\|_{\infty} \sup _{t \in\left(0, T_{0}\right)}\left\|\eta_{m}\right\|_{L^{2}\left(\mathbb{R}^{2}\right)} \\
& \quad \leq \varepsilon_{m} T_{0}\left\|\partial_{3} \phi_{3}\right\|_{\infty} \rightarrow 0, \quad \text { as } m \rightarrow \infty,
\end{aligned}
$$

we make use of (5.39) and (5.15) to obtain

$$
\begin{aligned}
& \left|\int_{0}^{T_{0}} \int_{\mathbb{R}^{2}}\left(\bar{\eta}_{m} \varphi_{m, 3}-\bar{\eta} \phi_{3}\left(t, x^{\prime}, 0\right)\right) \mathrm{d} x^{\prime} \mathrm{d} t\right| \\
& \quad \leq \int_{0}^{T_{0}} \int_{\mathbb{R}^{2}}\left|\left(\bar{\eta}_{m}-\bar{\eta}\right) \varphi_{m, 3}\right| \mathrm{d} x^{\prime} \mathrm{d} t+\int_{0}^{T_{0}} \int_{\mathbb{R}^{2}}\left|\bar{\eta}\left(\varphi_{m, 3}-\phi_{3}\left(t, x^{\prime}, 0\right)\right)\right| \mathrm{d} x^{\prime} \mathrm{d} t \\
& \quad \leq\left\|\phi_{3}\right\|_{\infty}\left\|\bar{\eta}_{m}-\bar{\eta}\right\|_{L^{1}\left((0, T) \times \mathcal{R}_{j_{3}}\right)}+\varepsilon_{m} T_{0}\left\|\partial_{3} \phi_{3}\right\|_{\infty} \rightarrow 0 \quad \text { as } m \rightarrow \infty,
\end{aligned}
$$

which gives

$$
\lim _{m \rightarrow \infty} \int_{0}^{T_{0}} \int_{\mathbb{R}^{2}} \bar{\eta}_{m} \varphi_{m, 3} \mathrm{~d} x^{\prime} \mathrm{d} t=\int_{0}^{T_{0}} \int_{\mathbb{R}^{2}} \bar{\eta} \phi_{3}\left(t, x^{\prime}, 0\right) \mathrm{d} x^{\prime} \mathrm{d} t .
$$

Here we have assumed that $\mathcal{R}_{j_{3}}$ satisfies

$$
\bigcup_{i=1}^{3} \bigcup_{t \in\left(0, T_{0}\right)} \operatorname{supp} \phi_{i}(t, x) \subset \mathcal{R}_{j_{3}}
$$

Combining (5.38) with (5.40), we arrive at 


$$
\lim _{m \rightarrow \infty} \int_{0}^{T_{0}} \int_{\Sigma_{m}(t)} \bar{\eta}_{m} \varphi_{m} \cdot \nu_{m} \mathrm{~d} S \mathrm{~d} t=\int_{0}^{T_{0}} \int_{\mathbb{R}^{2}} \bar{\eta} \phi_{3}\left(t, x^{\prime}, 0\right) \mathrm{d} x^{\prime} \mathrm{d} t .
$$

(ii) For the second term on the right-hand side of (5.32), taking into account that $\eta_{m}=\varepsilon \bar{\eta}_{m}$, we employ (5.33)-(5.36) to deduce that

$$
\begin{aligned}
& \int_{0}^{T_{0}} \int_{\Sigma_{m}(t)} \bar{H}_{m} \varphi_{m} \cdot \nu_{m} \mathrm{~d} S \mathrm{~d} t=-\sum_{i=1}^{2} \int_{0}^{T_{0}} \int_{\mathbb{R}^{2}}\left[\frac{\Delta_{x^{\prime}} \bar{\eta}_{m}+\left(\partial_{1} \eta_{m}\right)^{2} \partial_{2}^{2} \bar{\eta}_{m}+\left(\partial_{2} \eta_{m}\right)^{2} \partial_{1}^{2} \bar{\eta}_{m}}{\left(1+\left(\partial_{1} \eta_{m}\right)^{2}+\left(\partial_{2} \eta_{m}\right)^{2}\right)^{3 / 2}}\right. \\
& \left.\quad-\frac{2 \partial_{1} \eta_{m} \partial_{2} \eta_{m} \partial_{1} \partial_{2} \bar{\eta}_{m}}{\left(1+\left(\partial_{1} \eta_{m}\right)^{2}+\left(\partial_{2} \eta_{m}\right)^{2}\right)^{3 / 2}}\right] \varphi_{m, i} \partial_{i} \eta_{m} \mathrm{~d} x^{\prime} \mathrm{d} t \\
& \quad+\int_{0}^{T_{0}} \int_{\mathbb{R}^{2}} \frac{\left(\partial_{1} \eta_{m}\right)^{2} \partial_{2}^{2} \bar{\eta}_{m}+\left(\partial_{2} \eta_{m}\right)^{2} \partial_{1}^{2} \bar{\eta}_{m}-2 \partial_{1} \bar{\eta}_{m} \partial_{2} \eta_{m} \partial_{1} \partial_{2} \eta_{m}}{\left(1+\left(\partial_{1} \eta_{m}\right)^{2}+\left(\partial_{2} \eta_{m}\right)^{2}\right)^{3 / 2}} \varphi_{m, 3} \mathrm{~d} x^{\prime} \mathrm{d} t \\
& \quad+\int_{0}^{T_{0}} \int_{\mathbb{R}^{2}} \frac{\varphi_{m, 3} \Delta_{x^{\prime}} \bar{\eta}_{m}}{\left(1+\left(\partial_{1} \eta_{m}\right)^{2}+\left(\partial_{2} \eta_{m}\right)^{2}\right)^{3 / 2}} \mathrm{~d} x^{\prime} \mathrm{d} t,
\end{aligned}
$$

where the first two terms on the right-hand side can be estimated as follows, using the Sobolev imbedding theorem and (5.28), while the third term can be bounded below, following a procedure similar to that used for (5.39).

$$
\begin{aligned}
& \left|\int_{0}^{T_{0}} \int_{\mathbb{R}^{2}} \frac{\left(\partial_{1} \eta_{m}\right)^{2} \partial_{2}^{2} \bar{\eta}_{m}+\left(\partial_{2} \eta_{m}\right)^{2} \partial_{1}^{2} \bar{\eta}_{m}-2 \partial_{1} \bar{\eta}_{m} \partial_{2} \eta_{m} \partial_{1} \partial_{2} \eta_{m}}{\left(1+\left(\partial_{1} \eta_{m}\right)^{2}+\left(\partial_{2} \eta_{m}\right)^{2}\right)^{3 / 2}} \varphi_{m, 3} \mathrm{~d} x^{\prime} \mathrm{d} t\right| \\
& \leq\left\|\phi_{3}\right\|_{\infty} \int_{0}^{T_{0}} \int_{\mathcal{R}_{j_{3}}}\left|\left(\partial_{1} \eta_{m}\right)^{2} \partial_{2}^{2} \bar{\eta}_{m}+\left(\partial_{2} \eta_{m}\right)^{2} \partial_{1}^{2} \bar{\eta}_{m}-2 \partial_{1} \bar{\eta}_{m} \partial_{2} \eta_{m} \partial_{1} \partial_{2} \eta_{m}\right| \mathrm{d} x^{\prime} \mathrm{d} t \\
& \quad=\varepsilon_{m}^{2}\left\|\phi_{3}\right\|_{\infty} \int_{0}^{T_{0}} \int_{\mathcal{R}_{j_{3}}}\left|\left(\partial_{1} \bar{\eta}_{m}\right)^{2} \partial_{2}^{2} \bar{\eta}_{m}+\left(\partial_{2} \bar{\eta}_{m}\right)^{2} \partial_{1}^{2} \bar{\eta}_{m}-2 \partial_{1} \bar{\eta}_{m} \partial_{2} \bar{\eta}_{m} \partial_{1} \partial_{2} \bar{\eta}_{m}\right| \mathrm{d} x^{\prime} \mathrm{d} t \\
& \leq 4 \varepsilon_{m}^{2}\left\|\phi_{3}\right\|_{\infty} T_{0}\left\|\nabla \bar{\eta}_{m}\right\|_{L^{4}\left(\mathcal{R}_{j_{3}}\right)}^{2}\left\|\nabla^{2} \bar{\eta}_{m}\right\|_{L^{2}\left(\mathcal{R}_{j_{3}}\right)} \\
& \leq 4 \varepsilon_{m}^{2}\left\|\phi_{3}\right\|_{\infty} T_{0} c_{2}\left(j_{3}\right), \\
& \left|\int_{0}^{T_{0}} \int_{\mathbb{R}^{2}}\left[\frac{\Delta_{x^{\prime}} \bar{\eta}_{m}+\left(\partial_{1} \eta_{m}\right)^{2} \partial_{2}^{2} \bar{\eta}_{m}+\left(\partial_{2} \eta_{m}\right)^{2} \partial_{1}^{2} \bar{\eta}_{m}}{\left(1+\left(\partial_{1} \eta_{m}\right)^{2}+\left(\partial_{2} \eta_{m}\right)^{2}\right)^{3 / 2}} \frac{2 \partial_{1} \eta_{m} \partial_{2} \eta_{m} \partial_{1} \partial_{2} \bar{\eta}_{m}}{\left(1+\left(\partial_{1} \eta_{m}\right)^{2}+\left(\partial_{2} \eta_{m}\right)^{2}\right)^{3 / 2}}\right] \varphi_{m, i} \partial_{i} \eta_{m} \mathrm{~d} x^{\prime} \mathrm{d} t\right| \\
& \leq \varepsilon_{m}\|\phi\|_{\infty} \int_{0}^{T_{0}} \int_{\mathcal{R}_{j_{3}}}\left|\left(\Delta_{x^{\prime}} \bar{\eta}_{m}+\left(\partial_{1} \eta_{m}\right)^{2} \partial_{2}^{2} \bar{\eta}_{m}+\left(\partial_{2} \eta_{m}\right)^{2} \partial_{1}^{2} \bar{\eta}_{m}-2 \partial_{1} \eta_{m} \partial_{2} \eta_{m} \partial_{1} \partial_{2} \bar{\eta}_{m}\right) \partial_{i} \bar{\eta}_{m}\right| \mathrm{d} x^{\prime} \mathrm{d} t \\
& \leq \varepsilon_{m}\|\phi\|_{\infty} T_{0}\left(3 \varepsilon^{2}\left\|\nabla \bar{\eta}_{m}\right\|_{L^{6}\left(\mathcal{R}_{j_{3}}\right)}^{3}\left\|\nabla^{2} \bar{\eta}_{m}\right\|_{L^{2}\left(\mathcal{R}_{j_{3}}\right)}+\left\|\nabla \bar{\eta}_{m}\right\|_{L^{2}\left(\mathcal{R}_{j_{3}}\right)}\left\|\nabla^{2} \bar{\eta}_{m}\right\|_{L^{2}\left(\mathcal{R}_{j_{3}}\right)}\right) \\
& \leq \varepsilon_{m}\left\|\phi_{3}\right\|_{\infty} T_{0} c_{3}\left(j_{3}\right), \quad \varepsilon_{m}<3^{-1}, i=1,2
\end{aligned}
$$

where $c_{2}\left(j_{3}\right)$ and $c_{3}\left(j_{3}\right)$ are two constants depending on $j_{3}$, and

$$
\begin{aligned}
& \left|\int_{0}^{T_{0}} \int_{\mathbb{R}^{2}} \frac{\left(\varphi_{m, 3}-\phi_{3}\left(t, x^{\prime}, 0\right)\right) \Delta_{x^{\prime}} \bar{\eta}_{m}}{\left(1+\left(\partial_{1} \eta_{m}\right)^{2}+\left(\partial_{2} \eta_{m}\right)^{2}\right)^{3 / 2}} \mathrm{~d} x^{\prime} \mathrm{d} t\right| \\
& \leq T_{0} \sup _{t \in\left(0, T_{0}\right)}\left\|\Delta_{x^{\prime}} \bar{\eta}\right\|_{L^{2}\left(\mathbb{R}^{2}\right)}\left\|\partial_{3} \phi_{3}\right\|_{\infty} \sup _{t \in\left(0, T_{0}\right)}\left\|\eta_{m}\right\|_{L^{2}\left(\mathbb{R}^{2}\right)} \leq \varepsilon_{m} T_{0}\left\|\partial_{3} \phi\right\|_{\infty} \rightarrow 0 .
\end{aligned}
$$

On the other hand, applying (5.15) and the dominated convergence theorem, we conclude that

$$
\begin{aligned}
\left(1+\left(\partial_{1} \eta_{m}\right)^{2}+\left(\partial_{2} \eta_{m}\right)^{2}\right)^{-3 / 2} & =\left(1+\varepsilon_{m}^{2}\left(\partial_{1} \bar{\eta}_{m}\right)^{2}+\varepsilon_{m}^{2}\left(\partial_{2} \bar{\eta}_{m}\right)^{2}\right)^{-3 / 2} \\
& \rightarrow 1 \quad \text { strongly in } L^{2}\left(0, T_{0} ; L^{2}\left(\mathcal{R}_{j_{3}}\right)\right)
\end{aligned}
$$


as $m \rightarrow \infty$, while $\varepsilon_{m} \rightarrow 0$. Thus, from (5.46) and (5.14) we get

$$
\begin{aligned}
\lim _{m \rightarrow \int_{0}} \int_{\mathbb{R}^{2}} \frac{\phi_{3} \Delta_{x^{\prime}} \bar{\eta}_{m}}{\left(1+\left(\partial_{1} \eta_{m}\right)^{2}+\left(\partial_{2} \eta_{m}\right)^{2}\right)^{3 / 2}} \mathrm{~d} x^{\prime} \mathrm{d} t & =\lim _{m \rightarrow \int_{0}} \int_{\mathcal{R}_{j_{3}}}^{T_{0}} \frac{\phi_{3} \Delta_{x^{\prime}} \bar{\eta}_{m}}{\left(1+\left(\partial_{1} \eta_{m}\right)^{2}+\left(\partial_{2} \eta_{m}\right)^{2}\right)^{3 / 2}} \mathrm{~d} x^{\prime} \mathrm{d} t \\
& =\int_{0}^{T_{0}} \int_{\mathcal{R}_{j_{3}}} \frac{\phi_{3} \Delta_{x^{\prime}} \bar{\eta}}{\left(1+\left(\partial_{1} \eta\right)^{2}+\left(\partial_{2} \eta\right)^{2}\right)^{3 / 2}} \mathrm{~d} x^{\prime} \mathrm{d} t \\
& =\int_{0}^{T_{0}} \int_{\mathbb{R}^{2}} \frac{\phi_{3} \Delta_{x^{\prime}} \bar{\eta}}{\left(1+\left(\partial_{1} \eta\right)^{2}+\left(\partial_{2} \eta\right)^{2}\right)^{3 / 2}} \mathrm{~d} x^{\prime} \mathrm{d} t .
\end{aligned}
$$

In view of (5.45) and (5.47), we find that

$$
\lim _{m \rightarrow \infty} \int_{0}^{T_{0}} \int_{\mathbb{R}^{2}} \frac{\varphi_{m, 3} \Delta_{x^{\prime}} \bar{\eta}_{m}}{\left(1+\left(\partial_{1} \eta_{m}\right)^{2}+\left(\partial_{2} \eta_{m}\right)^{2}\right)^{3 / 2}} \mathrm{~d} x^{\prime} \mathrm{d} t=\int_{0}^{T_{0}} \int_{\mathbb{R}^{2}} \Delta_{x^{\prime}} \bar{\eta} \phi_{3}\left(t, x^{\prime}, 0\right) \mathrm{d} x^{\prime} \mathrm{d} t .
$$

Combining (5.43) with (5.44) and (5.48), we conclude that

$$
\lim _{m \rightarrow \infty} \int_{0}^{T_{0}} \int_{\Sigma_{m}(t)} \bar{H}_{m} \varphi_{m} \cdot \nu_{m} \mathrm{~d} S \mathrm{~d} t=\int_{0}^{T_{0}} \int_{\mathbb{R}^{2}} \Delta_{x^{\prime}} \bar{\eta} \phi_{3}\left(t, x^{\prime}, 0\right) \mathrm{d} x^{\prime} \mathrm{d} t .
$$

Consequently, it follows from (5.32), (5.37), (5.41) and (5.49) that

$$
\begin{aligned}
& \int_{0}^{T_{0}} \int_{\Omega} \varrho \partial_{t} \bar{u} \cdot \phi+\int_{0}^{T_{0}} \int_{\Omega}\left(\mu\left(\nabla \bar{u}+\nabla \bar{u}^{T}\right)-\bar{\sigma} I\right): \nabla \phi \mathrm{d} x \mathrm{~d} t \\
& \quad=g[\varrho] \int_{0}^{T_{0}} \int_{\mathbb{R}^{2}} \bar{\eta} \phi_{3}\left(t, x^{\prime}, 0\right) \mathrm{d} x^{\prime} \mathrm{d} t+\kappa \int_{0}^{T_{0}} \int_{\mathbb{R}^{2}} \Delta_{x^{\prime}} \bar{\eta} \phi_{3}\left(t, x^{\prime}, 0\right) \mathrm{d} x^{\prime} \mathrm{d} t .
\end{aligned}
$$

\subsection{Contradiction argument}

Similarly to (4.18), we multiply $(5.20)_{1}$ with $\phi \in\left(\mathcal{D}\left(\left(0, T_{0}\right) \times \Omega\right)\right)^{3}$ and integrate over $\left(0, T_{0}\right) \times \Omega$ to infer that

$$
\begin{aligned}
& \int_{0}^{T_{0}} \int_{\Omega} \varrho \partial_{t} \bar{u} \cdot \phi+\int_{0}^{T_{0}} \int_{\Omega}\left(\mu\left(\nabla \bar{u}+\nabla \bar{u}^{T}\right)-\bar{\sigma} I\right): \nabla \phi \mathrm{d} x \mathrm{~d} t \\
& =\int_{0}^{T_{0}} \int_{\mathbb{R}^{2}}\left(\left(\lambda_{+}(\bar{\sigma}) I-\mu_{+}\left(\nabla \bar{u}_{+}+\nabla \bar{u}_{+}^{T}\right)\right)-\left(\lambda_{-}(\bar{\sigma}) I-\mu_{-}\left(\nabla \bar{u}_{-}+\nabla \bar{u}_{-}^{T}\right)\right)\right) e_{3} \cdot \phi \mathrm{d} x^{\prime} \mathrm{d} t .
\end{aligned}
$$

Comparing (5.51) with (5.50), we get

$$
\begin{aligned}
\int_{0}^{T_{0}} & \int_{\mathbb{R}^{2}}\left(\left(\lambda_{+}(\bar{\sigma}) I-\mu_{+}\left(\nabla \bar{u}_{+}+\nabla \bar{u}_{+}^{T}\right)\right)-\left(\lambda_{-}(\bar{\sigma}) I-\mu_{-}\left(\nabla \bar{u}_{-}+\nabla \bar{u}_{-}^{T}\right)\right)\right) e_{3} \cdot \phi\left(t, x^{\prime}, 0\right) \mathrm{d} x^{\prime} \mathrm{d} t \\
& \left.=\int_{0}^{T_{0}} \int_{\mathbb{R}^{2}}\left(g[\varrho] \bar{\eta}+\kappa \Delta_{x^{\prime}} \bar{\eta}\right) e_{3} \cdot \phi\left(t, x^{\prime}, 0\right)\right) \mathrm{d} x^{\prime} \mathrm{d} t .
\end{aligned}
$$

On the other hand, by Lemma 4.1, (5.21), (5.11) and (4.11), we have

$$
\left(\lambda_{+}(\bar{\sigma}) I-\mu_{+}\left(\nabla \bar{u}_{+}+\nabla \bar{u}_{+}^{T}\right)\right)-\left(\lambda_{-}(\bar{\sigma}) I-\mu_{-}\left(\nabla \bar{u}_{-}+\nabla \bar{u}_{-}^{T}\right)\right) e_{3} \in L^{\infty}\left(0, T_{0} ;\left(L^{2}\left(\mathbb{R}^{2}\right)\right)^{3}\right),
$$

while by virtue of (5.28),

$$
g[\varrho] \bar{\eta}+\kappa \Delta_{x^{\prime}} \bar{\eta} \in L^{\infty}\left(0, T_{0} ; L^{2}\left(\mathbb{R}^{2}\right)\right) .
$$

Hence, by a density argument, we get from (5.52), (5.53) and (5.54) that

$$
\left[\left(\lambda_{+}(\bar{\sigma}) I-\mu_{+}\left(\nabla \bar{u}_{+}+\nabla \bar{u}_{+}^{T}\right)\right)-\left(\lambda_{-}(\bar{\sigma}) I-\mu_{-}\left(\nabla \bar{u}_{-}+\nabla \bar{u}_{-}^{T}\right)\right)\right] e_{3}=\left(g[\varrho] \bar{\eta}+\kappa \Delta_{x^{\prime}} \bar{\eta}\right) e_{3}
$$


holds a.e. in $\mathbb{R}^{2}$ and for a.e. $t \in\left(0, T_{0}\right)$.

In view of Definition 4.1, we find that $(\bar{\eta}, \bar{u})$ is just a strong solution of the linearized problem (1.5)-(1.7). By Remark 4.2, $(\tilde{\eta}, \tilde{u})$ is also a strong solution of $(1.5)-(1.7)$. Moreover, $\tilde{\eta}(0)=\bar{\eta}(0)$ and $\tilde{u}(0)=\bar{u}(0)$ (see $(5.23))$. Then, according to Theorem 4.1,

$$
\bar{u}=\tilde{u} \text { on }\left[0, T_{0}\right) \times \Omega .
$$

Hence, we may chain together the inequalities (5.21) and (5.2) to get

$$
2 \leq \sup _{T_{0} / 2 \leq t<T_{0}}\|\tilde{u}(t)\|_{H^{3}\left(\Omega_{ \pm}\right)} \leq \sup _{0 \leq t<T_{0}}\|\bar{u}\|_{H^{3}\left(\Omega_{ \pm}\right)} \leq 1
$$

which is a contraction. Therefore, the perturbed problem does not have the global stability of order $k$ for any $k \geq 3$. This completes the proof of Theorem 2.2.

Acknowledgements. This work was supported by the National Basic Research Program under the Grant 2011CB309705 and NSFC (Grant No. 40890154).

\section{References}

[1] R.A. Adams, J. John, Sobolev Space, Academic Press: New York, 2005.

[2] S. Chandrasekhar, Hydrodynamic and Hydromagnetic Stability, The International Series of Monographs on Physics, Oxford, Clarendon Press, 1961.

[3] R. Duan, F. Jiang, S. Jiang, On the Rayleigh-Taylor instability for incompressible, inviscid magnetohydrodynamic flows, submitted (2011).

[4] D. Ebin, The equations of motion of a perfect fluid with free boundary are not well posed, Comm. Partial Differential Equations 12 (1987) 1175-1201.

[5] D. Ebin, Ill-posedness of the Rayleigh-Taylor and Helmholtz problems for incompressible fluids, Comm. Partial Differential Equations 13 (1988) 1265-1295.

[6] Y. Guo, I. Tice, Compressible, inviscid Rayleigh-Taylor instability, arXiv:0911.4098v2 [math.AP] 23 Feb 2011, to appear in Indiana Univ. Math. J. (2011).

[7] Y. Guo, I. Tice, Decay of viscous surface waves without surface tension, arXiv:1011.5179v1 [math.AP] 23 Nov (2010).

[8] Y. Guo, I. Tice, Linear Rayleigh-Taylor instability for viscous, compressible fluids, SIAM J. Math. Anal. 42 (2011) 1688-1720.

[9] H.J. Hwang, Variational approach to nonlinear gravity-driven instability in a MHD setting, Quart. Appl. Math. 66 (2008) 303-324.

[10] H.J. Hwang, Y. Guo, On the dynamical Rayleigh-Taylor instability, Arch. Rational Mech. Anal. 167 (2003) 235-253.

[11] M. Kruskal, M. Schwarzschild, Some instabilities of a completely ionized plasma, Proc. Roy. Soc. (London) A 233 (1954) 348-360.

[12] G. Nespoli, R. Salvi, On the existence of two-phase problem for incompressible flow (ed. P. Maremonti), Quaderni di Mathematica 4 (1999). 
[13] A. Novotnỳ, I. Straškraba, Introduction to the Mathematical Theory of Compressible Flow, Oxford University Press, USA, 2004.

[14] L. Rayleigh, Analytic solutions of the Rayleigh equations for linear density profiles, Proc. London. Math. Soc. 14 (1883) 170-177.

[15] L. Rayleigh, Investigation of the character of the equilibrium of an in compressible heavy fluid of variable density, Scientific Paper, II (1990) 200-207.

[16] G.I. Taylor, The stability of liquid surface when accelerated in a direction perpendicular to their planes, Proc. Roy Soc. A 201 (1950) 192-196.

[17] J. Wang, Two-Dimensional Nonsteady Flows and Shock Waves (in Chinese), Science Press, Beijing, China, 1994.

[18] Y. Wang, Critical Magnetic number in the MHD Rayleigh-Taylor instability, arXiv: 1009.5422v1 [math.AP] 28 Sep 2010 (2010).

[19] J. Wehausen, E. Laitone, Surface Waves, Handbuch der Physik Vol. 9, Part 3, SpringerVerlag, Berlin, 1960. 